\title{
On the microwave optical properties of randomly oriented ice hydrometeors
}

\author{
P. Eriksson ${ }^{1}$, M. Jamali ${ }^{1,2}$, J. Mendrok ${ }^{2}$, and S. A. Buehler ${ }^{3}$ \\ ${ }^{1}$ Earth and Space Sciences, Chalmers University of Technology, 41296 Gothenburg, Sweden \\ ${ }^{2}$ Division of Space Technology, Lulea University of Technology, 98128 Kiruna, Sweden \\ ${ }^{3}$ Meteorological Institute, Center for Earth System Research and Sustainability, University of Hamburg, \\ Bundesstrasse 55, 20146 Hamburg, Germany \\ Correspondence to: P. Eriksson (patrick.eriksson@chalmers.se)
}

Received: 2 December 2014 - Published in Atmos. Meas. Tech. Discuss.: 21 December 2014

Revised: 31 March 2015 - Accepted: 11 April 2015 - Published: 5 May 2015

\begin{abstract}
Microwave remote sensing is important for observing the mass of ice hydrometeors. One of the main error sources of microwave ice mass retrievals is that approximations around the shape of the particles are unavoidable. One common approach to represent particles of irregular shape is the soft particle approximation (SPA). We show that it is possible to define a SPA that mimics mean optical particles of available reference data over narrow frequency ranges, considering a single observation technique at the time, but that SPA does not work in a broader context. Most critically, the required air fraction varies with frequency and application, as well as with particle size. In addition, the air fraction matching established density parameterisations results in far too soft particles, at least for frequencies above $90 \mathrm{GHz}$. That is, alternatives to SPA must be found.

One alternative was recently presented by Geer and Baordo (2014). They used a subset of the same reference data and simply selected as "shape model" the particle type giving the best overall agreement with observations. We present a way to perform the same selection of a representative particle shape but without involving assumptions on particle size distribution and actual ice mass contents. Only an assumption on the occurrence frequency of different particle shapes is still required. Our analysis leads to the same selection of representative shape as found by Geer and Baordo (2014). In addition, we show that the selected particle shape has the desired properties at higher frequencies as well as for radar applications.

Finally, we demonstrate that in this context the assumption on particle shape is likely less critical when using mass
\end{abstract}

equivalent diameter to characterise particle size compared to using maximum dimension, but a better understanding of the variability of size distributions is required to fully characterise the advantage.

Further advancements on these subjects are presently difficult to achieve due to a lack of reference data. One main problem is that most available databases of precalculated optical properties assume completely random particle orientation, while for certain conditions a horizontal alignment is expected. In addition, the only database covering frequencies above $340 \mathrm{GHz}$ has a poor representation of absorption as it is based on outdated refractive index data as well as only covering particles having a maximum dimension below $2 \mathrm{~mm}$ and a single temperature.

\section{Introduction}

Microwave techniques are gaining in importance for satellite observations of hydrometeors, i.e. clouds and precipitation. The main measurement target of microwave sensors is mass content estimates, possibly in the form of a precipitation rate. The detection mechanism used (absorption or scattering) depends on phase (liquid, ice or mixed), frequency and whether the instrument is active or passive. For example, for nonprecipitating liquid droplets passive measurements rely on absorption, while radars rely on backscattering. The signature of ice hydrometeors in passive data is a mix of scattering and absorption features, whereas in general the scattering part dominates (Sect. 4). 
The accuracy of the retrievals depends on technique applied and a number of variables, including observational noise and limitations in the radiative transfer code used. However, for ice mass retrievals the main retrieval error sources are frequently uncertainties associated with the microphysical state of the particles, i.e. phase, size, shape and orientation. This study focuses on the impact of assumed ice particle shape, probably the microphysical quantity with the least hope of being retrievable based on microwave data alone. Information on particle size can be obtained by combining data from different frequencies (Evans and Stephens, 1995a; Buehler et al., 2007; Jiménez et al., 2007), while the phase of the particles is largely determined by the atmospheric temperature. Measuring horizontal and vertical polarisation simultaneously reveals whether the particles have a tendency to horizontal alignment or their orientation is completely random (e.g. Hall et al., 1984; Hogan et al., 2003; Davis et al., 2005; Eriksson et al., 2011b).

Shape is normally not a critical aspect for purely liquid particles, as they are quasi-spherical throughout. The deviation from a strict spherical shape increases with droplet size and fall speed. However, the shape of frozen hydrometeors is highly variable, both as single crystals (needles, plates, columns, rosettes, dendrites, etc.) and as aggregates (see reviews by Heymsfield and McFarquhar, 2002; Baran et al., 2011). The shape is frequently denoted as the habit. It is unlikely that the air volume sampled contains a single ice particle shape, i.e. a habit mix can be expected. Furthermore, this mix normally varies with particle size. In principle, the shape of each particle should be known to avoid a related retrieval error, but this is not a feasible goal. Instead some "shape model" must be applied and the main aim of this study is to examine such models for microwave sounding of pure ice hydrometeors.

Considering the ice particles to be solid spheres is probably still the main microwave shape model. This approach is, for example, used in the standard 2B-CWC-O CloudSat retrievals by Austin et al. (2009). It is also applied in the Community Radiative Transfer Model (CRTM; Liu et al., 2013). Accordingly, retrieval systems (e.g. Boukabara et al., 2013; Gong and $\mathrm{Wu}, 2014$ ) and radiance assimilation based on CRTM inherit the assumption of solid spheres. Furthermore, this particle type has throughout been assumed in cloud ice retrievals based on limb sounding data (Wu et al., 2008; Rydberg et al., 2009; Millán et al., 2013). A main reason for the popularity of this shape model is that the single-scattering properties are simply calculated by wellestablished Mie codes.

Another common model is the "soft particle approximation" (SPA) where the particles are treated to consist of a homogeneous mix of ice and air. This approach requires that the volume or mass fraction of air and the corresponding refractive index of the ice-air mix are determined; see Sects. 2 and 5. SPA could in principle be used with a range of simplified particle forms, but it seems that only spheres and spheroids have been used so far. Spheroids are not treated by Mie theory but are covered by the also computationally efficient T-matrix method (Mishchenko et al., 1996). One application of SPA for practical retrievals is Zhao and Weng (2002). A more recent example is Hogan et al. (2012), arguing for using a soft spheroid model for cloud radar inversions. In addition, SPA has widely been used in studies to mimic measured radiances by radiative transfer tests (e.g. Bennartz and Petty, 2001; Skofronick-Jackson et al., 2002; Doherty et al., 2007; Meirold-Mautner et al., 2007; Kulie et al., 2010) in which the air fraction (AF) is either set to be fixed or derived from some parametric relationship between particle size and effective density.

Single-scattering properties for arbitrary particle shapes can be calculated e.g. by the discrete dipole approximation (DDA; Draine and Flatau, 1994). DDA is used for incorporating realistic particle shapes in the retrievals presented by Evans et al. (2012). This study is likely the most ambitious microwave retrieval set-up with regards to particle shape, but it deals only with a specific measurement campaign and it does not provide any general conclusions. Publicly available databases of DDA results for common particle shapes are reviewed in Sect. 3. These databases have been used in different ways: for example, Skofronick-Jackson et al. (2013) used one of the databases to estimate snowfall detection limits of several observation systems. Furthermore, the two main databases were used by Kulie et al. (2010) to test whether simulations could recreate some collocated radar and passive microwave data when applying different particle shapes. In a similar study by Geer and Baordo (2014), only passive data were considered but a more wide set of frequencies and atmospheric conditions were investigated. They found that a sector-like snowflake model gave the smallest overall error for the simulations performed. This choice will replace a SPA treatment as the default for the snow hydrometeor category in the RTTOV-SCATT (Bauer et al., 2006) package (Geer and Baordo, 2014). In Sect. 6 an alternative version of the approach of Geer and Baordo (2014) is tested that does not involve any assumption on particle size distribution (PSD) or actual ice masses.

DDA calculations have also been used in a more direct manner to investigate shape aspects. For example, Kim (2006) compared DDA and solid sphere results and claimed that particle shape is less critical for size parameters below 2.5 (see Eq. (2) for the definition), but only a few DDA shapes and frequencies were considered, radar backscattering was ignored and no quantitative error estimate was given. Comparisons between DDA and corresponding SPA data are found in e.g. Liu (2008), Nowell et al. (2013) and Liao et al. (2013), but the results have a limited scope throughout and we have found no comprehensive analysis of the limitations of SPA. An important result was obtained by Liu (2004), showing that an optimal "softness parameter", to be applied in a SPA framework, varies with frequency. The same con- 
clusion has also been reached in indirect ways by others, as pointed out by Geer and Baordo (2014).

From the perspective of mass retrievals it is most practical to characterise the size of the particles through their equivalent mass ice sphere diameter, $d_{\mathrm{e}}$ :

$d_{\mathrm{e}}=\sqrt[3]{\frac{6 m}{\rho_{i} \pi}}$,

where $m$ is the particle mass and $\rho_{i}$ is the density of (solid) ice. We define the size parameter, $x$, correspondingly:

$x=\frac{\pi d_{\mathrm{e}}}{\lambda}$,

where $\lambda$ is the wavelength at which the measurement is performed.

In microwave sounding, the mass is inferred from estimated extinction or backscattering coefficients. Any type of such coefficient, $\gamma$, can be expressed as

$\gamma=\int_{0}^{\infty} N\left(d_{\mathrm{e}}\right) \overline{\sigma_{\gamma}}\left(d_{\mathrm{e}}\right) \mathrm{d} d_{\mathrm{e}}$,

where $N\left(d_{\mathrm{e}}\right)$ is the PSD and $\overline{\sigma_{\gamma}}\left(d_{\mathrm{e}}\right)$ is the local average cross section for particles having a mass matching $d_{\mathrm{e}}$. In its turn, Eq. (3) implies that trying to estimate $\overline{\sigma_{\gamma}}\left(d_{\mathrm{e}}\right)$ from observed satellite data (as done in Kulie et al., 2010; Geer and Baordo, 2014) requires a good knowledge of both the mass of frozen hydrometeors and the PSD.

Another common way to express particle size is by the maximum diameter, $d_{\mathrm{m}}$. We start the study by using $d_{\mathrm{e}}$, because usage of $d_{\mathrm{m}}$ demands that the relation between $d_{\mathrm{m}}$ and particle mass must be introduced. Such relationships depend on particle shape, and for the basic purpose of this study that is a problematic complication. By using $d_{\mathrm{e}}$, particle shape only influences $\overline{\sigma_{\gamma}}\left(d_{\mathrm{e}}\right)$. However, as $d_{\mathrm{m}}$ is probably more frequently used than $d_{\mathrm{e}}$, this alternative to characterise particle sizes is the last step of the study (Sect. 7).

In summary, our scope is the approximation of particle shape in microwave retrievals of the mass of pure ice hydrometeors. Focus is put on SPA and the basic conclusion of Geer and Baordo (2014). Both passive and active measurements are considered, because merging information from different sensor types is already in use (e.g. Rydberg et al., 2009; Kulie et al., 2010), and such synergies should in the future just grow in importance. The practical aim can be seen as finding a shape model that gives a good estimate of $\overline{\sigma_{\gamma}}\left(d_{\mathrm{e}}\right)$, for relevant optical properties, over a large range of particle sizes, frequencies, measurement techniques and possible habit mixes. Existing DDA data are reviewed and used as reference. Only complete random orientation is treated because most established publicly available DDA databases are currently limited to this assumption on orientation.

Furthermore, compared to earlier similar works, much higher attention is given to frequencies above $200 \mathrm{GHz}$.
Ice mass retrievals are already performed at sub-millimetre wavelengths using limb sounding (Wu et al., 2008; Rydberg et al., 2009; Millán et al., 2013) and airborne sensors (Evans et al., 2012). Additionally, a strong motivation for this assessment is upcoming sub-millimetre instruments: the European ISMAR (International Sub-Millimetre Airborne Radiometer) airborne instrument and the ICI (Ice Cloud Imager) sensor will be part of the next series of Metop satellites. ICI is a down-looking sub-millimetre cloud ice sensor, a concept that has already been described in several articles (Evans and Stephens, 1995a; Buehler et al., 2007, 2012) but for which so far no actual satellite sensor has been available. This study is part of our overall effort to build the scientific foundation for the analysis of first the ISMAR airborne and then eventually the ICI satellite data.

\section{Refractive index}

Any calculation of single-scattering properties, i.e. independently whether Mie, T-matrix or DDA calculations are performed, requires that the refractive index is specified. Parameterisations and expressions related to the refractive index of ice at microwave frequencies are reviewed in this section. Both the real $\left(n^{\prime}\right)$ and imaginary $\left(n^{\prime \prime}\right)$ part of the complex refractive index $n$ are relevant. Some relationships are more easily expressed in terms of the (relative, complex) dielectric constant, $\epsilon$. Neglecting magnetic effects, which is a good assumption here, this quantity is related to the (complex) refractive index as

$n=\sqrt{\epsilon}$.

\subsection{Pure ice models}

Providing complex refractive index practically over the complete electromagnetic spectrum in the form of data tables, Warren (1984), in the following referred to as W84, has been a long-term standard in atmospheric science for the refractive index of pure water ice. Hufford (1991, H91) developed a parameterisation for microwave frequencies up to $1 \mathrm{THz}$ based on Debye and Lorenz theories with parameters fitted from measured data. The parameterisation was incorporated in the MPM93 atmospheric propagation model by Liebe et al. (1993). Compared to W84, H91 generally predicts lower $n^{\prime \prime}$ for frequencies $<350 \mathrm{GHz}$ (see Fig. 1, right panel). Consistent with measurements it predicts a stronger increase of $n^{\prime \prime}$ with temperature than W84 at sub-millimetre frequencies.

In the advent of sub-millimetre observation techniques, Jiang and $\mathrm{Wu}$ (2004, J04) added a higher-order frequency term to H91, as suggested by Mishima et al. (1983), in order to cover frequencies up to $3 \mathrm{THz}$. Resulting $n^{\prime \prime}$ agree well with $\mathrm{H} 91$ until about $1 \mathrm{THz}$, as shown in Fig. 1. Beyond $1 \mathrm{THz}$, where H91 claims no validity, J04's $n^{\prime \prime}$ exhibits the frequency and temperature dependence pattern expected from measured far-infrared behaviour of $n^{\prime \prime}$. Zhang et al. (2001, Z01) did the first measurements of both $n^{\prime}$ and $n^{\prime \prime}$ 

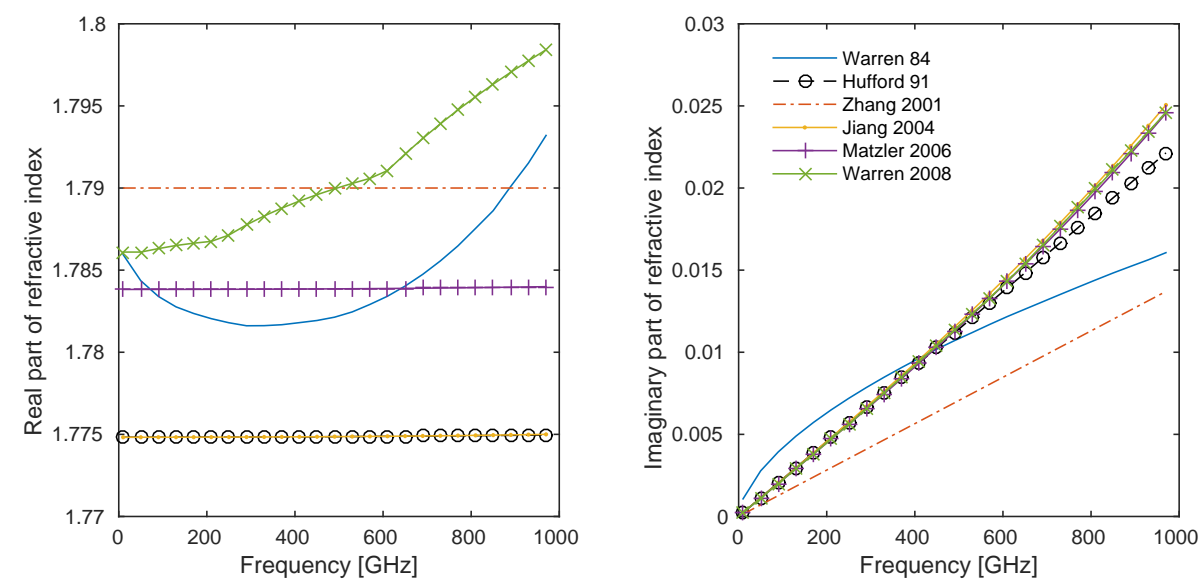

Figure 1. Real (left) and imaginary (right) part of the refractive index of pure ice as a function of frequency, according to Warren (1984), Hufford (1991), Zhang et al. (2001), Jiang and Wu (2004), Mätzler (2006) and Warren and Brandt (2008). The temperature is set to 266 K.

at sub-millimetre frequencies and atmospheric temperatures. For $n^{\prime \prime}$ they found a linear temperature dependence of about $1 \% \mathrm{~K}^{-1}$. The measurements agree quite well with the $\mathrm{H} 91$ and J04 models. However, the Z01 model falls short of reproducing their own measurements - it predicts very low values over all frequencies (see Fig. 1) and all temperatures.

Mätzler (2006, M06) introduced a permittivity parameterisation that consolidates most earlier models and measurements. Regarding the imaginary part, it agrees well with the J04 model, particularly at microwave frequencies, deviating by less than $5 \%$ at higher frequencies and high temperatures. The largely revised and updated version of W84, the Warren and Brandt (2008, W08) data, incorporates the M06 model at $T=-7^{\circ} \mathrm{C}$ and proposes M06 as the model of choice at wavelengths beyond $200 \mu \mathrm{m}$ when temperature dependence should be considered.

J04 found their model to be within $12 \%$ of the imaginary permittivity measurements for frequencies below $800 \mathrm{GHz}$ and within $15-40 \%$ above $800 \mathrm{GHz}$. M06 estimated the uncertainty of their model from the standard deviations of measurements to $5 \%$ at $270 \mathrm{~K}$ and $14 \%$ at $200 \mathrm{~K}$. W08 state the uncertainty of their $n^{\prime \prime}$ data to be $10 \%\left(T=-7^{\circ} \mathrm{C}\right)$. Compared to recent models, the once quasi-standard model W84 strongly overestimates $n^{\prime \prime}$ at millimetre wavelengths (up to a factor of 5), underestimates it at sub-millimetre wavelengths (up to a factor of 2) and overestimates it in the far-infrared (up to a factor of 2). The impact on particle absorption due to selection of M06 or W84 is exemplified below (Sect. 3.4).

In contrast to $n^{\prime \prime}, n^{\prime}$ is generally considered to be known with higher accuracy and to vary little to negligibly with both temperature and frequency. The measurements by Z01 confirm the small frequency dependence $(0.3 \%$ over 250 $1000 \mathrm{GHz})$ and do not show significant temperature dependence. The different models mentioned above provide slightly different relations of $n^{\prime}$ with frequency and temperature. However, we find refractivity $\left(n^{\prime}-1\right)$ from all models to agree with M06 within $1.3 \%$, according to the left panel of Fig. 1. Based on estimates on propagation of $n^{\prime}$ uncertainty to optical properties, we conclude that the uncertainty in $n^{\prime}$ is not a limiting factor and choice of model is not critical.

In summary, M06 seems the best choice for microwave to far-infrared imaginary refractive index data. In view of the effects that errors in imaginary refractive index have on cloud optical properties (see Sect. 3.4), we strongly suggest no longer using the Warren (1984) data in future.

\subsection{Mixing rules}

The parameterisations reviewed above deal with solid ice, while in the soft particle approximation (Sect. 5) the particles are treated to consist of a homogeneous mixture of ice and air. The standard procedure for assigning a refractive index to the mixture is to apply a so-called mixing rule. In this paper we compare some commonly used mixing rules from a purely practical perspective; a more theoretical review of mixing rules is provided by Sihvola (2000).

Throughout we will assume the refractive index of air to be $1+i 0$; in other words we assume that the optical properties of air are like those of a vacuum. Of course, for the radiative transfer problem as a whole both absorption and refraction by air matter strongly. However, given the much larger refractive index of ice we neglect this in the calculation of the singlescattering properties, as commonly done by other authors.

Three mixing rules are considered: "Maxwell-Garnett" (Garnett, 1906), "Bruggeman" (Bruggeman, 1935) and "Debye" (Debye, 1929). All these formulas operate with dielectric constants. The Debye mixing rule is

$\frac{\epsilon_{\mathrm{e}}-1}{\epsilon_{\mathrm{e}}+2}=\frac{f_{1}^{\mathrm{v}}\left(\epsilon_{1}-1\right)}{\epsilon_{1}+2}+\frac{\left(1-f_{1}^{\mathrm{v}}\right)\left(\epsilon_{2}-1\right)}{\epsilon_{2}+2}$,

where $\epsilon_{\mathrm{e}}$ is the "effective" dielectric constant of the mixture, $f_{1}^{\mathrm{v}}$ is the volume fraction of medium $1\left(f_{1}^{\mathrm{v}}+f_{2}^{v}=1\right)$ and $\epsilon_{1}$ 

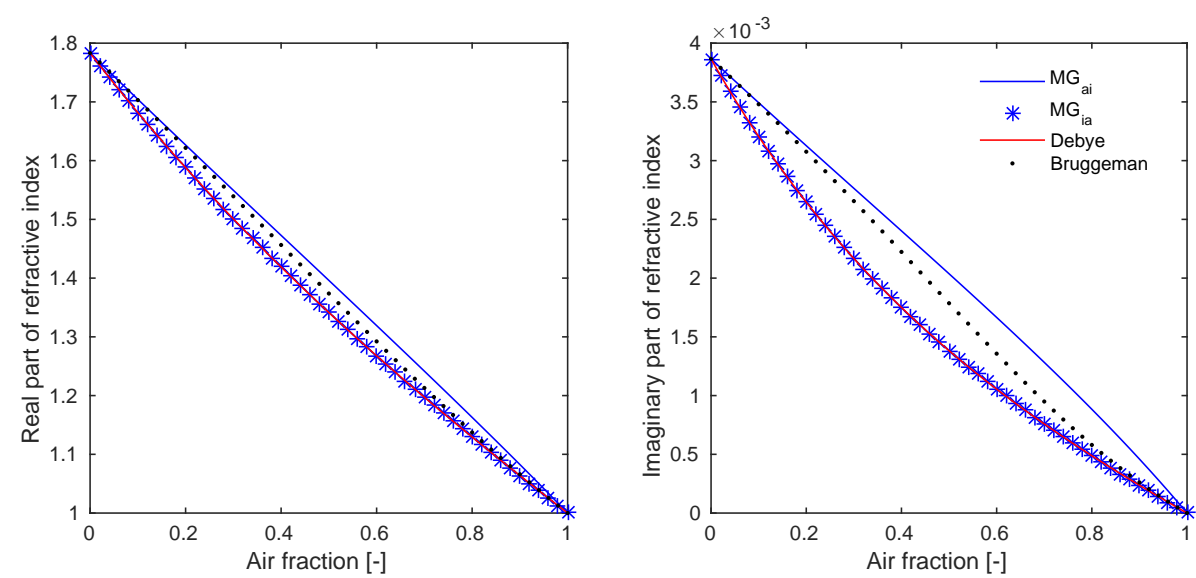

Figure 2. Real (left) and imaginary (right) part of the effective refractive index of a mixture of ice and air as a function of air volume fraction according to some mixing rules. The refractive index of ice at $183 \mathrm{GHz}$ and $263 \mathrm{~K}, n_{\text {ice }}=1.7831+i 0.0039$, was used, and the refractive index of air was set to $n_{\text {air }}=1+i 0$.

and $\epsilon_{2}$ are $\epsilon$ for medium 1 and 2 respectively. The expression for Bruggeman is

$\frac{f_{1}^{\mathrm{v}}\left(\epsilon_{1}-\epsilon_{\mathrm{e}}\right)}{\epsilon_{1}+2 \epsilon_{\mathrm{e}}}+\frac{\left(1-f_{1}^{\mathrm{v}}\right)\left(\epsilon_{2}-\epsilon_{\mathrm{e}}\right)}{\epsilon_{2}+2 \epsilon_{\mathrm{e}}}=0$

The Debye and Bruggeman expressions are symmetric with respect to the two media. Maxwell-Garnett differs in this respect by making a distinction between the "matrix" $\left(\epsilon=\epsilon_{\mathrm{m}}\right)$ and the "inclusion" $\left(\epsilon=\epsilon_{\mathrm{i}}\right)$ :

$\epsilon_{\mathrm{e}}=\epsilon_{\mathrm{m}}+3 f_{\mathrm{i}}^{\mathrm{v}} \epsilon_{\mathrm{m}} \frac{\left(\epsilon_{\mathrm{i}}-\epsilon_{\mathrm{m}}\right)}{\epsilon_{\mathrm{i}}+2 \epsilon_{\mathrm{m}}-f_{\mathrm{i}}^{\mathrm{v}}\left(\epsilon_{\mathrm{i}}-\epsilon_{\mathrm{m}}\right)}$,

where is $f_{\mathrm{i}}^{\mathrm{v}}$ is the volume fraction of the inclusion medium $\left(f_{m}^{v}+f_{\mathrm{i}}^{\mathrm{v}}=1\right)$. That is, for Maxwell-Garnett we have two cases, "air in ice" and "ice in air" (below shortened to $\mathrm{MG}_{\mathrm{ai}}$ and $\mathrm{MG}_{\mathrm{ia}}$ respectively), that result in different $\epsilon_{\mathrm{e}}$ depending on whether air is set to be the matrix or inclusion medium.

For completeness, the effective density $\left(\rho_{e}\right)$ matching $f_{1}^{\mathrm{v}}$ is

$\rho_{e}=f_{1}^{\mathrm{v}} \rho_{1}+\left(1-f_{1}^{\mathrm{v}}\right) \rho_{2}$,

where $\rho_{1}$ and $\rho_{2}$ are the density of medium 1 and 2 respectively. In terms of mass fraction of medium $1\left(f_{1}^{\mathrm{m}}\right), \rho_{e}$ is

$\rho_{e}=\frac{\rho_{1} \rho_{2}}{f_{1}^{\mathrm{m}} \rho_{2}+\left(1-f_{1}^{\mathrm{m}}\right) \rho_{1}}$.

An example comparison between the mixing rules is shown in Fig. 2. A first observation is that the Debye and the "ice in air" version of Maxwell-Garnett $\left(\mathrm{MG}_{\mathrm{ia}}\right)$ give identical results (for $\epsilon_{\mathrm{m}}=\epsilon_{\text {air }}=1+i 0$ the two formulas are mathematically identical). Hence, the Debye rule is not explicitly discussed below but is represented by the identical $\mathrm{MG}_{\mathrm{ia}} \cdot \mathrm{MG}_{\mathrm{ia}}$ gives consistently the lowest refractive index for both real and imaginary part. The difference from the other two rules is highest at air fractions around 0.45 . The highest values are throughout found for $\mathrm{MG}_{\mathrm{ai}}$, and Bruggeman falls between the two Maxwell-Garnett versions. Repeating the calculations for other frequencies and temperatures (e.g. Johnson et al., 2012, Fig. 2), i.e. other ice refractive indices, shows that these patterns are of general validity and are not specific to our example.

The deviations between the mixing rules are significant. For example, Johnson et al. (2012) conducted a sensitivity analysis for frequencies between 2.8 and $150 \mathrm{GHz}$ regarding the choice of mixing rule. The differences when using $\mathrm{MG}_{\mathrm{ai}}$ or $\mathrm{MG}_{\mathrm{ia}}$ were found to be $\sim 2 \mathrm{~dB}$ for radar reflectivity and reach at least $10 \mathrm{~K}$ for brightness temperature.

Some mixing rule can be optimal for representing a true homogeneous ice-air spherical particle, as studied by Petty and Huang (2010), but this is not the crucial point in this context. In Sect. 5.1 we instead pragmatically test whether any of the mixing rules leads to a simpler approximation of realistically shaped particles.

\section{Existing DDA databases}

The DDA is the most widely used method for computing the scattering properties of arbitrarily shaped particles. In the DDA method, a particle is represented by an array of dipoles in a cubic lattice with a given inter-dipole spacing. This spacing must be adequately small relative to the incident wavelength in order to obtain desired accuracy, which requires large computer memory and long calculation time for large particles.

Despite the wide usage of the method, the publicly available DDA data for microwave scattering of ice particles are limited. The three databases that are used in this study are the ones of Liu (2008), Hong et al. (2009) and Nowell et al. (2013). The main properties of these databases are summarised in Table 1. 
Table 1. Overview of considered DDA databases.

\begin{tabular}{|c|c|c|c|c|}
\hline Database & $\begin{array}{c}\text { Frequency range } \\
{[\mathrm{GHz}]}\end{array}$ & $\begin{array}{c}\text { Temperatures } \\
{[\mathrm{K}]}\end{array}$ & $\begin{array}{c}\text { Particle sizes } \\
{[\mu \mathrm{m}, \text { max. dim. }]}\end{array}$ & Particle shapes \\
\hline Liu (2008) & $3.0-340$ & $\begin{array}{c}233,243,253 \\
263 \text { and } 273\end{array}$ & $50-12454$ & $\begin{array}{l}\text { Columns, plates, rosettes, } \\
\text { sector and dendrite snowflakes }\end{array}$ \\
\hline Nowell et al. (2013) & $10.65-183.31$ & 263 & $200-12584$ & $\begin{array}{l}\text { Three aggregate types, } \\
\text { consisting of } 200 \text { and/or } 400 \mu \mathrm{m} \\
\text { six-bullet rosettes }\end{array}$ \\
\hline Hong et al. (2009) & $90-874$ & 243 & $2-2000$ & $\begin{array}{l}\text { Solid and hollow columns, } \\
\text { plates, six-bullet rosettes, } \\
\text { droxtals and one type of aggregate }\end{array}$ \\
\hline
\end{tabular}

The only other open source of microwave DDA data that we know about is http://helios.fmi.fi/ tyynelaj/, where data used in Tyynelä and Chandrasekar (2014) and some other publications were recently made available. These data, covering frequencies up to $220 \mathrm{GHz}$, are not included in this paper as they deal with partly oriented particles, while the other databases all are valid for completely random orientation.

\subsection{Liu}

Liu (2008) applied the DDA code of Draine and Flatau (2000), denoted as DDSCAT, and computed single-scattering properties (i.e. scattering cross section, absorption cross section, backscattering cross section, asymmetry parameter and phase function) of 11 types of ice particle crystal shapes, at 22 frequencies $(3,5,9,10,13.4,15,19,24.1,35.6,50,60$, $70,80,85.5,90,94,118,150,166,183,220$ and $340 \mathrm{GHz}$ ) and for five different temperatures. To not clutter the figures below, we include only 6 of the 11 particle types. The ignored shapes are: short column, block column, thin plate and four- and five-bullet rosettes; included shapes are pointed out in the figure legends. The included six particle types cover the full range of variation in the optical properties found in the database.

The particles were treated to have random orientation. The phase function is provided for 37 equally spaced scattering angles between 0 and $180^{\circ}$. In terms of the "phase matrix" required for vector radiative transfer, only the $(1,1)$ element is given. The refractive index of ice applied in the DDA calculation was taken from Mätzler (2006).

\subsection{Nowell}

A new snowflake aggregation model is introduced in Nowell et al. (2013). The six-bullet rosette is a frequently observed crystal shape and therefore was selected by Nowell et al. (2013) as constituent crystals of the simulated snowflake aggregates. The aggregates were allowed to grow in three dimensions, following an algorithm resulting in quasi-spherical snowflakes following the diameter-density parameterisation of Brandes et al. (2007). The representation of the bullet rosettes is somewhat coarse, based on cubic blocks with a size of $\approx 50 \mu \mathrm{m}$. Only particles with a maximum diameter above $1 \mathrm{~mm}$ are included in our figures to make sure that the aggregates consist of a relatively high number of building blocks.

The single-scattering properties of an ensemble of randomly generated aggregates were calculated by the DDSCAT code. Calculations for 10 frequencies $(10.65,13.6,18.7$, $23.8,35.6,36.5,89,94,165.5$ and $183.31 \mathrm{GHz})$ and a single temperature $(263 \mathrm{~K})$ were performed, with refractive index taken from Mätzler (2006). The phase function is not included in this database; only the corresponding asymmetry parameter is stored.

\subsection{Hong}

Hong et al. (2009) also used DDSCAT to compute the scattering properties (extinction efficiency, absorption efficiency, single-scattering albedo, asymmetry parameter and scattering phase matrix) of six randomly oriented non-spherical ice particles at 21 frequencies $(90,118,157,166,183.3,190$, 203, 220, 243, 325, 340, 380, 425, 448, 463, 487, 500, 640, 664,683 and $874 \mathrm{GHz}$ ) for a temperature of $243 \mathrm{~K}$. All six independent elements of the phase matrix are reported in steps of $1^{\circ}$ between 0 and $180^{\circ}$.

The geometrical information of the six ice particle shapes is detailed in Table 1 of Hong (2007). Refractive index of ice was taken from Warren (1984), which according to Sect. 2.1 is not the optimal choice with respect to particle absorption.

\subsection{Comparison of the databases}

Example DDA data are found in Fig. 3 for one of the few frequencies that is found in all three databases $(183 \mathrm{GHz})$. All three aggregate types in the Nowell database are plotted with the same symbol. The abscissa of the figure is size parameter according to Eq. (2), implying that the radiative properties are compared between particles having the same mass. Absorption, scattering and backscattering are reported 

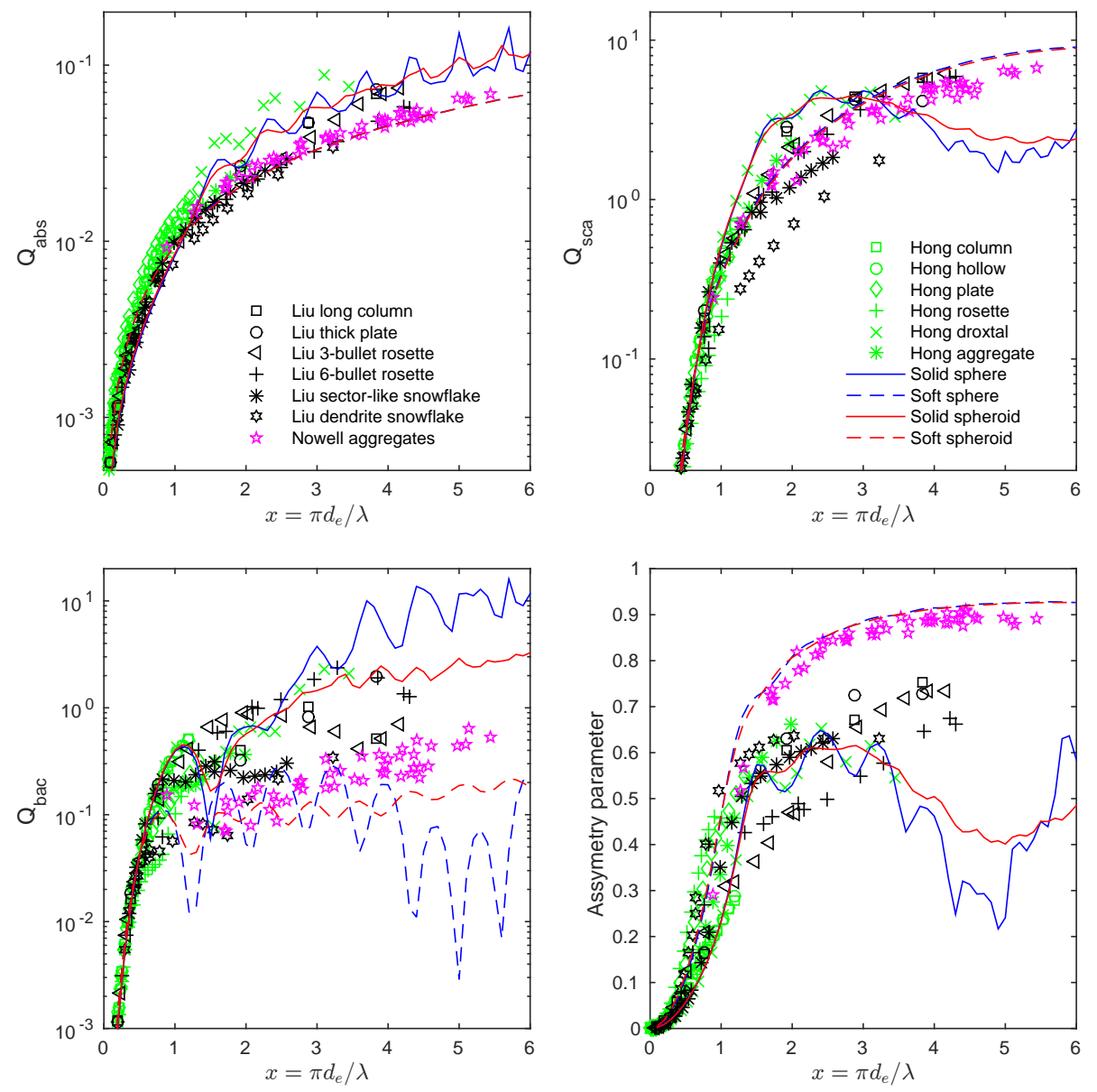

Figure 3. DDA-based single-scattering properties at $183 \mathrm{GHz}$ from the databases of Liu (2008), Nowell et al. (2013) and Hong et al. (2009). Absorption, scattering and backscattering efficiencies (Eq. 10) and asymmetry parameter are displayed. The combined legends are valid for all panels. The figure includes also data of solid and soft spheres and spheroids, with refractive index following Mätzler (2006). The soft particles have an air fraction of 0.75 , with the effective refractive indices derived by the $\mathrm{MG}_{\mathrm{ai}}$ mixing rule. The spheroids are oblate with an aspect ratio of 1.67. All results are valid for $183 \mathrm{GHz}$ and $243 \mathrm{~K}$ except those of Nowell et al. (2013) for $263 \mathrm{~K}$.

as the corresponding efficiency, $Q$, calculated with respect to $d_{\mathrm{e}}$ as

$Q=\frac{4 \sigma}{\pi d_{\mathrm{e}}^{2}}$,

where $\sigma$ is the cross section of concern. Even though usage of $Q$ provides some normalisation of the data, compared to when cross sections were to be plotted, the ordinates in the first three panels of Fig. 3 still span several orders of magnitude. In Fig. 4 another normalisation is applied that brings out differences at lower size parameters: the optical cross sections are divided by the corresponding optical cross section of the equivalent mass solid ice sphere with same ice refractive index.

Although we discuss the soft particle approximation in depth only in Sect. 5, solid and soft spheroids are already included in the figures here for reference. We also already make some remarks on their optical properties here but post- pone the explanation of how the soft-spheroid results were generated to the dedicated section later.

The Hong data have systematically a higher absorption than Liu. This can be discerned in Fig. 3 and is expected due to the higher imaginary part of the refractive index $\left(n^{\prime \prime}\right)$ in W84 (used by Hong) compared to M06 (used by Liu) for frequencies below $400 \mathrm{GHz}$, as shown in Fig. 1. This deviation is removed in Fig. 4 as the normalisation is done with respect to Mie calculations with the refractive index set to match the DDA data. Without this adjustment of the refractive index there would be a much higher variability in the absorption ratios in Fig. 4, with the different DDA databases at different mean levels. Droxtal particles are quasi-spherical and the fact that these particles obtain ratios very close to 1 in Fig. 4 confirms that a correct normalisation has been applied. This similarity in shape explains also why the droxtals end up close to the data for solid spheres for all quantities in Fig. 3. 

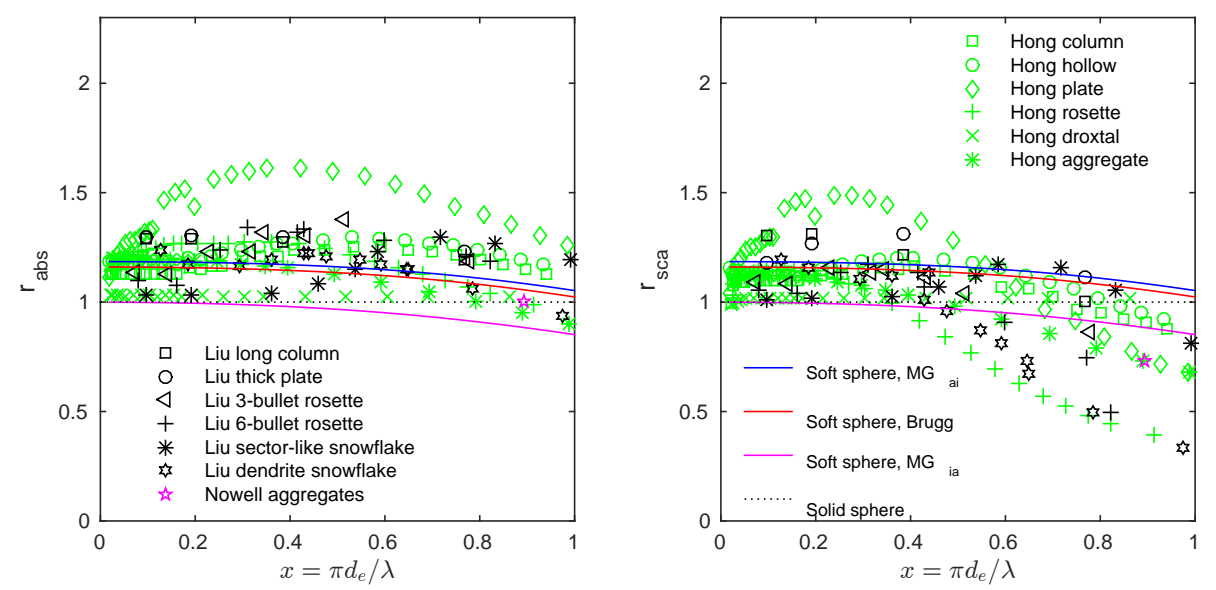

Figure 4. Absorption (left) and scattering (right) cross sections of DDA data and soft spheres at $183 \mathrm{GHz}$. The cross sections are reported as the ratio to the corresponding cross section of the equivalent mass sphere, with the same refractive index as used for the preparation of the DDA data. That is, the dotted straight line at $r=1$ represents solid ice spheres. Database source and particle shapes of the DDA data are found in figure legends (same as in Fig. 3). The soft spheres have an air fraction of 0.25, where results for three different mixing rules (MG ${ }_{i a}$, Bruggeman and $\mathrm{MG}_{\mathrm{ia}}$ ) are included (solid lines).

It is well known that the impact of shape on the extinction efficiency increases with particle size. Accordingly, for $x$ below $\sim 0.5$ there is a comparably low spread between different particles for both absorption and scattering. In terms of the ratio in Fig. 4, the data are mainly inside 1.2 \pm 0.2 .

However, at $x=2$ the difference between the lowest and highest scattering, for particles having the same mass, is about a factor of five (Fig. 3). These remarks consider also $340 \mathrm{GHz}$ (not shown) where the same particles result in higher size parameters.

The backscattering efficiency shows a similar pattern as the scattering one, but the variation above $x=2$ is considerably higher by about a factor of 10 . This is the case because the backscattering depends on the phase function for a particular direction, resulting in a higher sensitivity to the exact shape of that function, while the overall scattering extinction corresponds to the integrated phase function.

There is a significant spread in the asymmetry parameter ( $g$ ) from about $x=0.5$ and above. Above $x \approx 1.5$, the difference between highest and lowest $g$ is about 0.3 , where the Nowell aggregates and the Liu bullet rosettes throughout cause the highest and lowest values respectively. The sixbullet rosettes in the Hong database show the same tendency of low $g$ for combinations of size and frequency resulting in $x>1.5$. At lower $x$ the Hong rosettes tend to give the highest $g$ among all of the particles, then also higher than the corresponding Liu rosette. That is, the different six-bullet rosette models used by Liu and Hong result in significantly different optical properties.

Figure 3 was inspired by Fig. 7 of Nowell et al. (2013), comparing that database with solid and soft particle calculations in the same way. Nowell et al. (2013) used a higher air fraction for their soft particles and it is not clear whether the
$\mathrm{MG}_{\mathrm{ai}}$ or $\mathrm{MG}_{\mathrm{ia}}$ version of the Maxwell-Garnett mixing rule was used, but there are still some clear deviations between the two figures for soft particles. For example, the scattering efficiency of soft particles in our Fig. 3 is quite close to the data from Nowell et al. (2013), while in their Fig. 7 the soft particles give significantly lower scattering. In addition, we obtain basically identical scattering efficiencies for soft spheres and spheroids, while Nowell et al. (2013) got lower scattering for spheroids. We have carefully checked our calculations and our results seem to fit better with what has been found elsewhere. For example, in Fig. 5 of Liao et al. (2013) a good agreement with the aggregates of Nowell et al. (2013) is obtained by soft particles having a density of $0.2 \mathrm{~g} \mathrm{~m}^{-3}$ (the air fraction of 0.75 in Fig. 3 matches $0.23 \mathrm{~g} \mathrm{~m}^{-3}$ ), and basically identical results are obtained between spheres and both prolate and oblate spheroids.

\section{Relevance of absorption and asymmetry parameter}

To judge the performance of a soft particle approximation or some "shape model", a basic consideration is the detail in which the single-scattering properties must be compared. The main issue is that the phase function (describing the angular redistribution of scattered radiation, also denoted as the scattering function) can be very complex and is basically unique for all particles where Rayleigh conditions do not apply. However, it is normally not required to compare the phase function in full detail. For example, it is in general only the direct backscattering that is of interest for radar applications. This is valid until multiple scattering becomes significant, when also the phase function starts to be relevant.

For passive measurements, the standard choice is to give an overall description of the phase function by using the parameter $g$. The asymmetry parameter is known to have a 


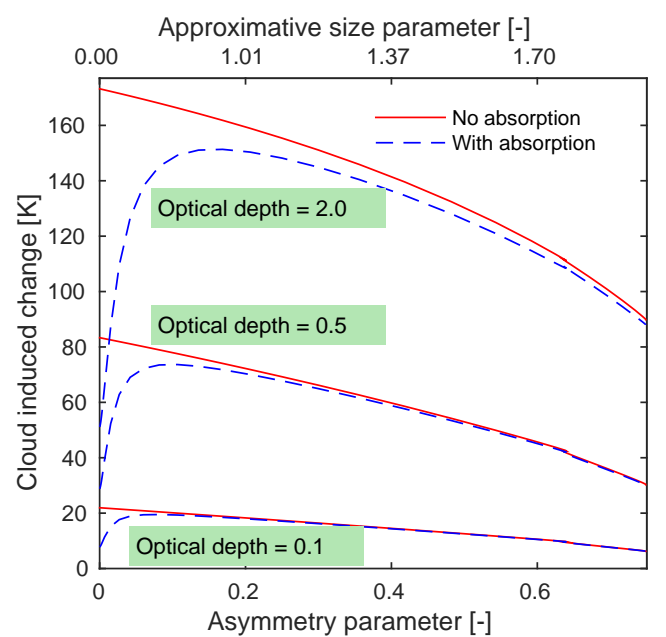

Figure 5. Test of importance of absorption and asymmetry parameter for passive microwave radiative transfer. The brightness temperature deviation from simulations with no cloud layer is reported, where a positive value in the figure corresponds to a decrease in absolute brightness temperature. The stated optical depths refer to the zenith extinction of the cloud layer. For solid lines, the imaginary part of the refractive index was set to 0 , resulting in no cloud particle absorption. The simulations are described further in the text.

strong influence in radiative transfer of solar radiation (e.g. Kahnert et al., 2008). The quantity is also frequently reported in connection to passive microwave radiative transfer (e.g. Liu, 2004; Kim, 2006), but, to our best knowledge, the actual influence of $g$ for such applications has not been investigated in a general manner. A simple test of this type is found in Fig. 5. The calculations were done with the DOIT (Discrete Ordinate ITerative method) scattering module of the ARTS radiative transfer model (Emde et al., 2004; Eriksson et al., 2011a). Satellite measurements at $150 \mathrm{GHz}$ and an incidence angle of $45^{\circ}$ were simulated. Temperature and gas profiles were taken from a standard tropical scenario (Fascod), and a $2 \mathrm{~km}$ thick "cloud" layer, centred at $10 \mathrm{~km}$, was added. The selection of surface emission is not critical for these qualitative calculations and for simplicity the surface was treated to act as a blackbody. A single particle size (monodispersive PSD) was used for each simulation, and the number of particles was adjusted to obtain the specified zenith optical depths. Spherical particles with an intermediate air fraction (0.4) were assumed, and $d_{\mathrm{e}}$ was varied to obtain a range of $g$. Solid ice particles could not be used for this test as they don not give a monotonic increase of $g$ with particle size, and neither provide $g$ above 0.7 (Fig. 3).

The solid lines in Fig. 5 show how the brightness temperature changes with $g$ when the cloud optical depth is kept constant and all particle absorption is suppressed. The basic pattern is that the cloud impact on measured radiance decreases with increasing $g$. This makes sense as high $g$ means that the up-welling emission from the lower troposphere is less redi- rected compared to the case of more isotropic scattering at low $g$; see Buehler et al. (2007) for a schematic figure and discussion of the radiative transfer for this measurement geometry. It is hard to see in the figure, but there actually are some "wiggles" around $g=0.65$, showing that the relationship to $g$ is not completely monotonic. That is, several values of $g$ can result in the same radiance.

In Fig. 5 the cloud impact for $g=0$ and $g=0.6$ differs by a factor of about 2 . That is, changing $g$ by 0.1 results in $\mathrm{a} \sim 10 \%$ change in cloud impact. For low optical thickness the relationship between scattering cross section $\left(\sigma_{\mathrm{s}}\right)$ and radiance impact is close to linear. Accordingly, a $10 \%$ error in $\sigma_{\mathrm{s}}$ and a 0.1 error in $g$ are in rough terms equally important. The test displayed in Fig. 5 was repeated for other frequencies and cloud altitudes. The absolute values of the cloud impact change, primarily following the magnitude of the gas absorption at the altitudes around the cloud layer, but the mentioned relation between $\sigma_{\mathrm{s}}$ and $g$ was found to be relatively constant.

Figure 5 exemplifies also the importance of ice particle absorption for passive measurements. It is well known that absorption is most significant for smaller particles, i.e. the single-scattering albedo increases with particle size (e.g. Evans and Stephens, 1995b; Eriksson et al., 2011b). Figure 5 confirms this as the difference between considering absorption (dashed lines) and neglecting it (solid lines) is high for small $x$ for all cloud optical depths. This aspect is especially important for limb sounding, because in this observation geometry focus is put on higher altitudes where smaller particles are more frequent, and it has been shown that the measured signal can even be dominated by absorption (Wu et al., 2014).

Figure 5 also shows the less obvious fact that absorption increases in importance with increasing cloud optical thickness. For an optical thickness of 2.0 absorption is significant up to at least $x=1.2$, while for small optical depths (such as 0.1 ) the absorption can be neglected for $x$ above $\sim 0.5$. This is a consequence of the behaviour that probability of absorption increases when multiple scattering becomes more prominent. The changed conditions caused by multiple scattering implies that the relevance of absorption can not be judged alone from the single-scattering albedo parameter. In addition, the observation geometry matters for the relative importance of absorption and scattering, as discussed in Eriksson et al. (2011b).

In summary, it is confirmed that the quantities normally considered $\left(\sigma_{a}, \sigma_{\mathrm{s}}, \sigma_{b}\right.$ and $\left.g\right)$ are all relevant but to a varying degree. Most importantly, the relevance of absorption decreases with size parameter.

\section{Approximation by soft particles}

The soft particle approach (SPA) is based on two main simplifications. Firstly, the particle is treated to consist of a homogeneous mix of air, ice and, when mixed-phase particles 

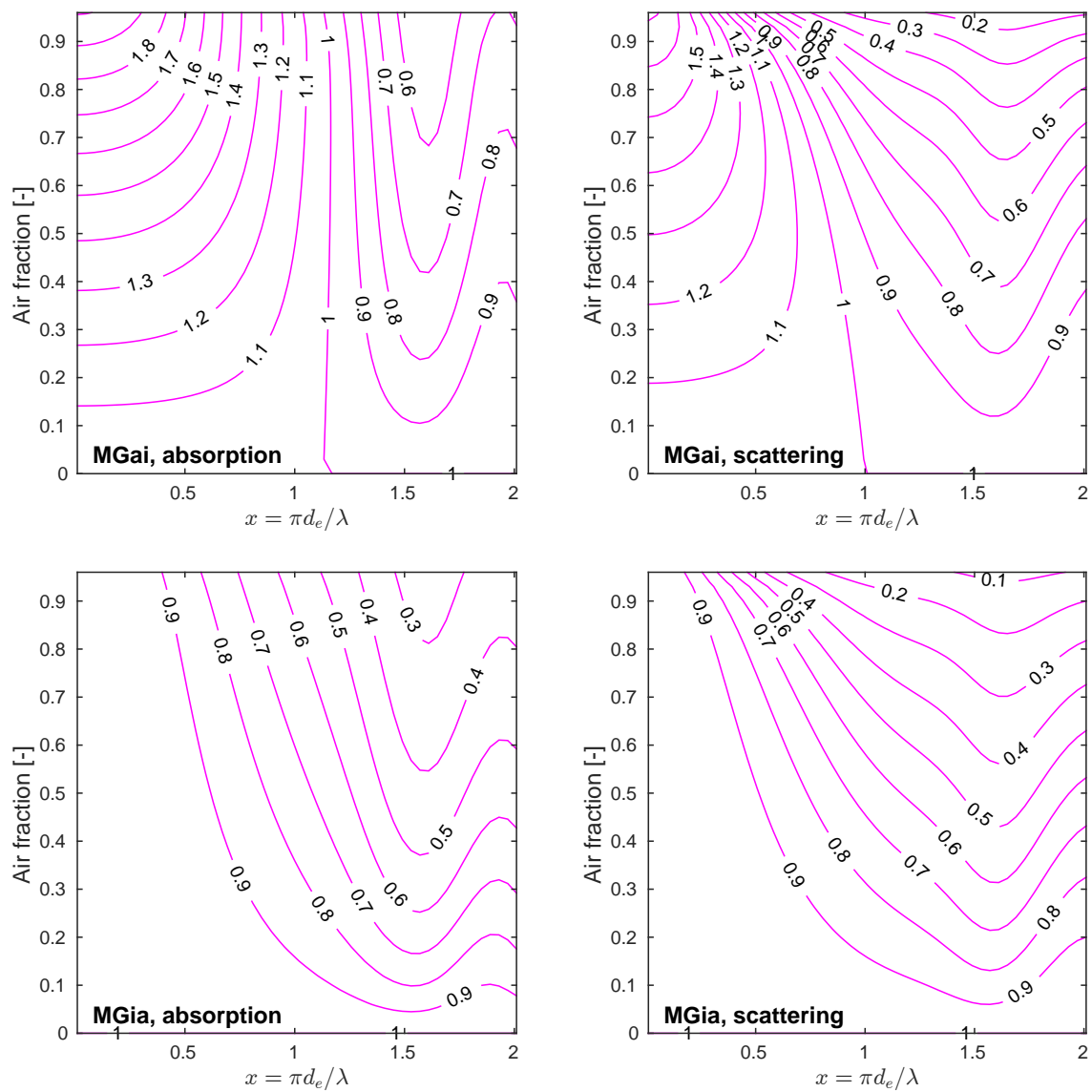

Figure 6. Absorption (left) and scattering (right) cross sections of soft spheres ( $183 \mathrm{GHz}$ and $243 \mathrm{~K}$ ), normalised by the equivalent mass ice sphere absorption or scattering cross section as in Fig. 4, as a function of size parameter and air fraction. The two top panels are calculated using the $\mathrm{MG}_{\mathrm{ai}}$ (air in ice) mixing rule, while the two lower panels are calculated using the $\mathrm{MG}_{\mathrm{ia}}$ (ice in air) mixing rule.

are considered, also water (e.g. Galligani et al., 2013). The air fraction of the mix is either set to a constant value or is obtained by assuming an effective density of the particle, likely varying with particle maximum size. A single refractive index is assigned to the mix by applying a mixing rule (Sect. 2.2). Secondly, the particles must be set to have some specific shape, to allow the single-scattering properties to be determined with a limited calculation burden. As mentioned, the T-matrix method allows e.g. soft columns and plates to be possible options, but the standard choices are to model the particles as spheres or spheroids. A much more detailed description of SPA is provided by Liao et al. (2013).

\subsection{Selection of mixing rule}

As a first step, we examined whether the choice of mixing rule is critical in any way for SPA. The difference between mixing rules can in general be compensated by selecting different air fractions, but exceptions exist. This is most clearly seen for the absorption and scattering cross section at smaller $x$, as exemplified in Fig. 4. In the figure, the absorption of soft particles when using Maxwell-Garnet with "ice in air"
$\left(\mathrm{MG}_{\mathrm{ia}}\right)$ is throughout lower than the DDA results. This is in contrast to using the Bruggeman or the "air in ice" version of Maxwell-Garnett mixing rule $\left(\mathrm{MG}_{\mathrm{ai}}\right)$, in which the soft particle absorption matches some of the DDA data points. The same pattern is found also for the scattering cross section but for a smaller range of $x$.

The low bias in Fig. 4 of $\mathrm{MG}_{\mathrm{ia}}$, compared to DDA data, can not be removed by modifying the air fraction, as shown in Fig. 6. In this figure, the ratios of Fig. 4 are calculated for air fractions between 0 and 0.95 and the ratios obtained when using $\mathrm{MG}_{\mathrm{ia}}$ are below 1 throughout. Ratios around at least 1.2 are required to represent the average values of the DDA data in Fig. 4. For $x<0.5$ such ratios, and even much higher values, can be obtained by selecting the $\mathrm{MG}_{\mathrm{ai}}$ mixing rule. Ratios when using Bruggeman (not shown) reach 1.25 for absorption and 1.15 for scattering, which is on the limit to fit the DDA data. For $\mathrm{MG}_{\mathrm{ai}}$ the ratios switch from being $>1$ to $<1$ around $x=1$, which in the following is shown to be the general behaviour of DDA data.

The conclusion of Figs. 4 and 6 is that using the $\mathrm{MG}_{\mathrm{ia}}$ mixing rule leads to a systematic underestimation of the absorption and scattering at some size parameter ranges. The 
same applies to the Debye mixing rule as it is identical to $\mathrm{MG}_{\mathrm{ia}}$. The Bruggeman rule gives higher values but is not capable of reproducing the highest DDA-based ratios found in Fig. 4. The patterns seen in Fig. 6 are not specific to $183 \mathrm{GHz}$ but are representative of the complete microwave region (1 to $1000 \mathrm{GHz}$ ). In addition, these remarks do not depend on whether soft spheres or spheroids are used. Accordingly, $\mathrm{MG}_{\mathrm{ai}}$ appears as the best choice in this context, and only this mixing rule is considered below.

\subsection{Selection of particle shape}

Solid spheres are known to exhibit resonance features for $x$ above $\sim 1$, which are reflected as oscillations in the properties displayed in Fig. 3 (blue solid lines). An individual spheroid would give similar oscillations, but the assumption of completely random orientation partly averages out those patterns for the spheroids (see Fig. 3, red solid lines). The resonance phenomena are dampened when going to soft particles. This results in a marginal difference in extinction (absorption and scattering cross sections) and $g$ between soft spheres and spheroids (dashed lines). However, there is a significant difference for the backscattering, where soft spheres give even stronger oscillations than solid spheres. The soft spheroids show a more smooth variation with $x$, and should allow a better fit to the DDA data. Liao et al. (2013) made the same observations for soft particles and showed that extinction and $g$ are basically unaffected by the aspect ratio of the spheroids or whether they are oblate or prolate. They found also that the oscillations in size dependence of the backscattering decrease when the aspect ratio moves away from 1.

That is, soft spheroids are preferable over soft spheres, primarily due to the difference with respect to backscattering. For complete random orientation the selection of aspect ratio is not critical, and only oblate spheroids with an axial ratio of 0.6 are considered below, following Hogan et al. (2012). In terms of the nomenclature used in the T-matrix code, this equals an aspect ratio of 1.67 .

\subsection{Selection of air fraction}

As shown in the above figures, for a given size parameter there is a spread of the particle optical properties over the different habits. Hence, it is not possible to match all particle shapes at the same time with a soft particle approximation. The ambition is instead to approximately mimic the average properties. Figure 3 shows that solid spheres and spheroids do not meet this criterion because they (e.g. for $x>4$ ) systematically underestimate scattering cross section and $g$.

It is stressed that in this work, air fraction is treated as a pure tuning parameter. This implies that statements about low/high air fractions just refer to values relatively close to $0 / 1$; they are not judgements about the true density of the particles.

\subsubsection{Relevance of the reference data}

A more detailed analysis requires some consideration of the occurrence frequency of the different particles in the DDA databases. For example, the Hong database contains droxtals having a maximum diameter, $d_{\mathrm{m}}$, up to $2 \mathrm{~mm}$, while the general view is that this shape is only representative for the smallest ice crystals (Baran, 2012). In fact, Schmitt and Heymsfield (2014) found that cloud ice particles with $d_{\mathrm{m}}$ above $250 \mu \mathrm{m}$ are mainly the aggregate type, implying that also single plates and columns having dimensions above this size are relatively rare. The aggregate types discussed by Schmitt and Heymsfield (2014) appear to be relatively similar to the aggregates in the Hong database.

For the representation of particles of snow type, the Liu database offers two shapes (dendrite and sector-like snowflakes) that both have high aspect ratios, while the aggregates of the Nowell database (claimed to represent snowflakes) have an aspect ratio close to 1 . This difference in aspect ratio results particularly in deviations in the scattering cross section and $g$ of those particles (Fig. 3). If "snow" is understood as everything from the classical single-crystal snowflake to graupel, both these assumptions on aspect ratio are realistic, but it is clear that particles having intermediate aspect ratios also exist and, hence, they are not yet covered by the DDA databases. The Hong aggregates could potentially also work as proxy for snow particles, but data for $d_{\mathrm{m}}$ above $2 \mathrm{~mm}$ are lacking.

\subsubsection{Fit of single-scattering data}

Figure 3 exemplifies a fit of the available DDA data using soft particles having an AF of 0.75 . For the frequency of concern $(183 \mathrm{GHz})$ the soft particles, compared to the solid ones, give indeed a better general fit of absorption and scattering efficiencies. For $g$ at $x>1.5$, the soft particles agree well with the Nowell aggregates, while all other particle shapes are better approximated with solid spheres or a comparably low AF. That is, for e.g. the Liu sector-like snowflake, the AF that gives the best fit with scattering efficiency is not the same AF that is needed to match $g$.

Example results for other frequencies are found in Fig. 7. This figure, together with Fig. 4 covering $183 \mathrm{GHz}$, indicate that the $\mathrm{MG}_{\mathrm{ai}}$ mixing rule combined with an $\mathrm{AF}$ of 0.25 give an acceptable fit of absorption and scattering for size parameters below 0.5, independent of frequency. At higher $x$, $\mathrm{AF}=0.25$ is not optimal; it gives a fairly good fit at $874 \mathrm{GHz}$ (Fig. 7), but at e.g. $90 \mathrm{GHz}$ this AF results in too-high values for both absorption and scattering. For $x>1$ and lower frequencies, a fit of absorption and scattering efficiencies requires higher AFs. For example, mimicking the scattering efficiency at $90 \mathrm{GHz}$ requires an AF on the order of 0.75-0.9, depending on whether all or just snow-type particles should be fitted. 

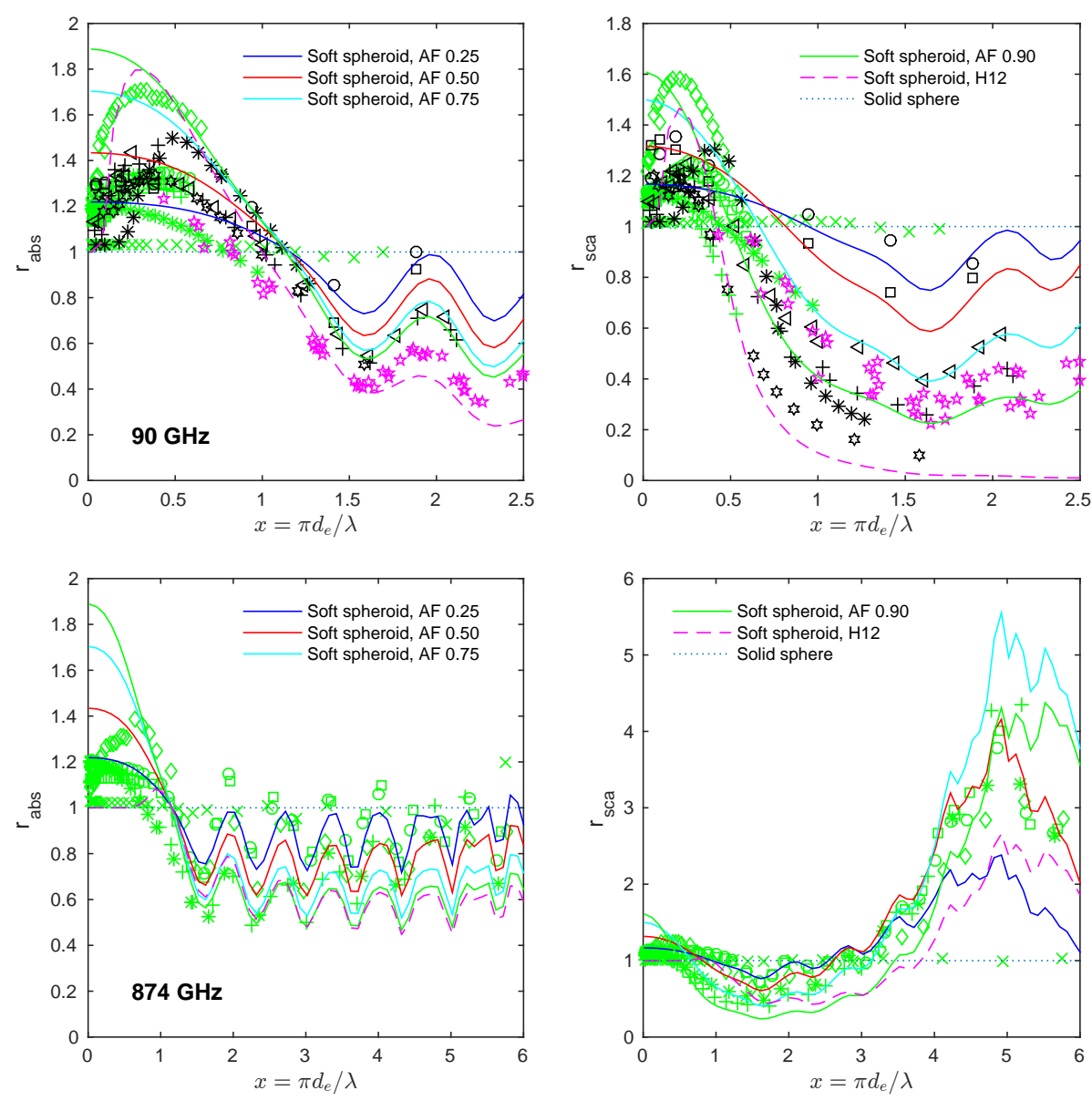

Figure 7. Normalised absorption (left) and scattering (right). The top row includes $90 \mathrm{GHz}$ Hong/Liu and $89 \mathrm{GHz}$ Nowell data, while the bottom row covers $874 \mathrm{GHz}$. The soft spheroids have either a fixed air fraction (AF) or follow Hogan et al. (2012), denoted as H12. Normalisation and plotting symbols used for DDA data are as in Fig. 4.

These remarks show three facts. Firstly, there is in general not a single AF that simultaneously gives a fit of all four optical property parameters. Secondly, at least when operating at lower frequencies, the AF to apply in a soft particle approximation must be allowed to vary with particle size. Thirdly, the best AF has a frequency dependence (at least for larger $x$ ). The third point is well known and has been shown by e.g. Liu (2004).

The soft particle AF is frequently set to follow some density parameterisation. This gives the $\mathrm{AF}$ a variation with size in line with the second point. However, the known fact that an optimal AF varies with frequency (point 3) signifies that using true densities cannot work as a general approach with respect to optical properties. This is the case because densitybased AFs are independent of frequency. In addition, for larger particles standard density parameterisations result in much higher AFs than the ones giving a match of singlescattering data around $100 \mathrm{GHz}$ and above. As an example, the particle model of Hogan et al. (2012) is included in Fig. 7. This particle model is based on the frequently used density parameterisation of Brown and Francis (1995) that leads to AFs close to 1 for the largest DDA particles. In fact, the scattering efficiency at $90 \mathrm{GHz}$ becomes too low already at $x \approx 0.5$. Also, backscattering is underestimated at $x$ above $\approx 0.5$ even at lower frequencies (Fig. 8). All other density parameterisations we have tested show the same general feature: to produce, in this context, too-high AFs for larger particles. For more recent parameterisations the density goes below $100 \mathrm{~kg} \mathrm{~m}^{-3}$ at $d_{\mathrm{m}} \approx 800 \mu \mathrm{m}$ (Cotton et al., 2013, Fig. 6).

Hogan et al. (2012) was selected as it provides a clearly defined particle model. However, it should be noted that Hogan et al. (2012) treat the spheroids to be aligned with the maximum dimension in the horizontal plane, while we apply completely random orientation.

\subsubsection{Test radiative transfer simulations}

Absorption, scattering and asymmetry parameter interact for simulations of passive observations, as shown in Sect. 4. Some test simulations were performed in order to check whether the different tendencies for these quantities combine 

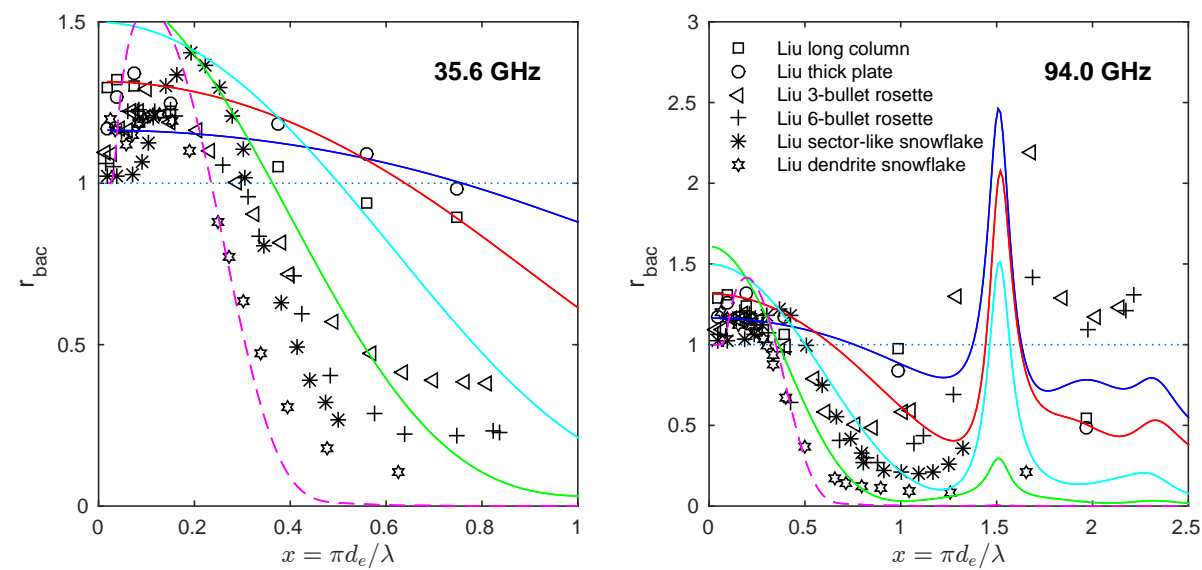

Figure 8. Normalised backscattering of the Liu particles at two frequencies. As in Figs. 4 and 7, the normalisation is performed with respect to the backscattering cross section of the solid sphere having the same mass. For $94 \mathrm{GHz}$ and $x \approx 1.5$ some data points have a ratio above 3 partly due to a minimum of the solid sphere backscattering at that size parameter. Solid and dotted lines are the same as in Fig. 7.

in a positive or negative manner. These simulations, shown in Fig. 9, were performed for the same scenario as used for Fig. 5. Again, a monodispersive PSD was used, but here the number of particles was adjusted to obtain a specified vertical column of ice mass, or ice water path (IWP). The IWP for each frequency was selected to give a maximum cloudinduced brightness temperature change of $5-10 \mathrm{~K}$ in order to get a significant response but still avoid a high degree of multiple scattering.

As mentioned, the radiative transfer simulations were done with ARTS-DOIT (Sect. 4). This scattering method requires the full phase function and no results for the Nowell database could be generated. For Fig. 9 a more strict selection of the DDA particles was done, roughly matching the discussion in Sect. 5.3.1 in order to remove the less physically plausible particles. Column, plates and three-bullet rosettes having $d_{\mathrm{m}}$ above $1 \mathrm{~mm}$ were excluded. For droxtals the limit was set to $200 \mu \mathrm{m}$. The black solid lines show a polynomial fit (in linear scale) of the simulations based on the remaining DDA particles.

As expected from the discussion above, the different DDA particles give little spread of simulated brightness temperatures for $x<0.5$. However, there is a strong variation at larger size parameters. For example, the Liu dendrite snowflakes, as well the Hong six-bullet rosettes as around $x=1$, have particularly low influences. This is a combined effect of relatively low scattering efficiency and high $g$ (Fig. 4). The same combination enhances also the differences between the Liu sector-like and dendrite snowflakes compared to the differences for scattering efficiency alone. The relative influence between the particles is not the same for all frequencies. For example, the Hong aggregates are found on the high side for 90 and $166 \mathrm{GHz}$ but are rather on the low side for $874 \mathrm{GHz}$.
Figure 9 shows that the selection of the soft particle AF is not highly critical for size parameters below 0.5 . This is partly due to compensating errors. A too-high AF gives an overestimation of both absorption and scattering, but this is counteracted by an overestimation of $g$. At higher size parameters, the frequency dependence of the "optimal" AF noted above is also seen here. For example, at $340 \mathrm{GHz}$ an AF of $0.25-0.50$ is required to match the fit of the DDA results (black line), while for $90 \mathrm{GHz}$ a suitable $\mathrm{AF}$ is above 0.75 . For $874 \mathrm{GHz}$, only covered by the Hong database, an $\mathrm{AF}$ around 0.25 gives best agreement. The systematic deviation between the soft particle and the DDA-based results seen for $874 \mathrm{GHz}$ and low $x$ is due to the refractive index differences discussed in Sect. 2.1.

\section{Approximation by a single representative shape}

Based on poor experience of using the SPA at ECMWF (the European Centre for Medium-Range Weather Forecasts), Geer and Baordo (2014) attacked the representation of particle shape in microwave radiative transfer from another angle. Their application is data assimilation for numerical weather prediction, but the basic problem is the same as for direct retrieval of frozen hydrometeors. Their approach is simple: to try to find a particle type, for which DDA calculations are at hand, that minimises the average deviation to actual observations. They compared to measurements from the TMI and SSMIS sensors for frequencies between 10 and $190 \mathrm{GHz}$. The Hong database does not cover the lower end of this frequency range, and only the Liu database was considered.

They performed global simulations for latitudes between $60^{\circ} \mathrm{S}$ and $60^{\circ} \mathrm{N}$. Simulated brightness temperatures were obtained with RTTOV-SCATT, a radiative transfer tool making use of several approximations. The atmospheric data were taken from the ECMWF 4D-Var assimilation system, likely having biases in ice mass amounts varying between 

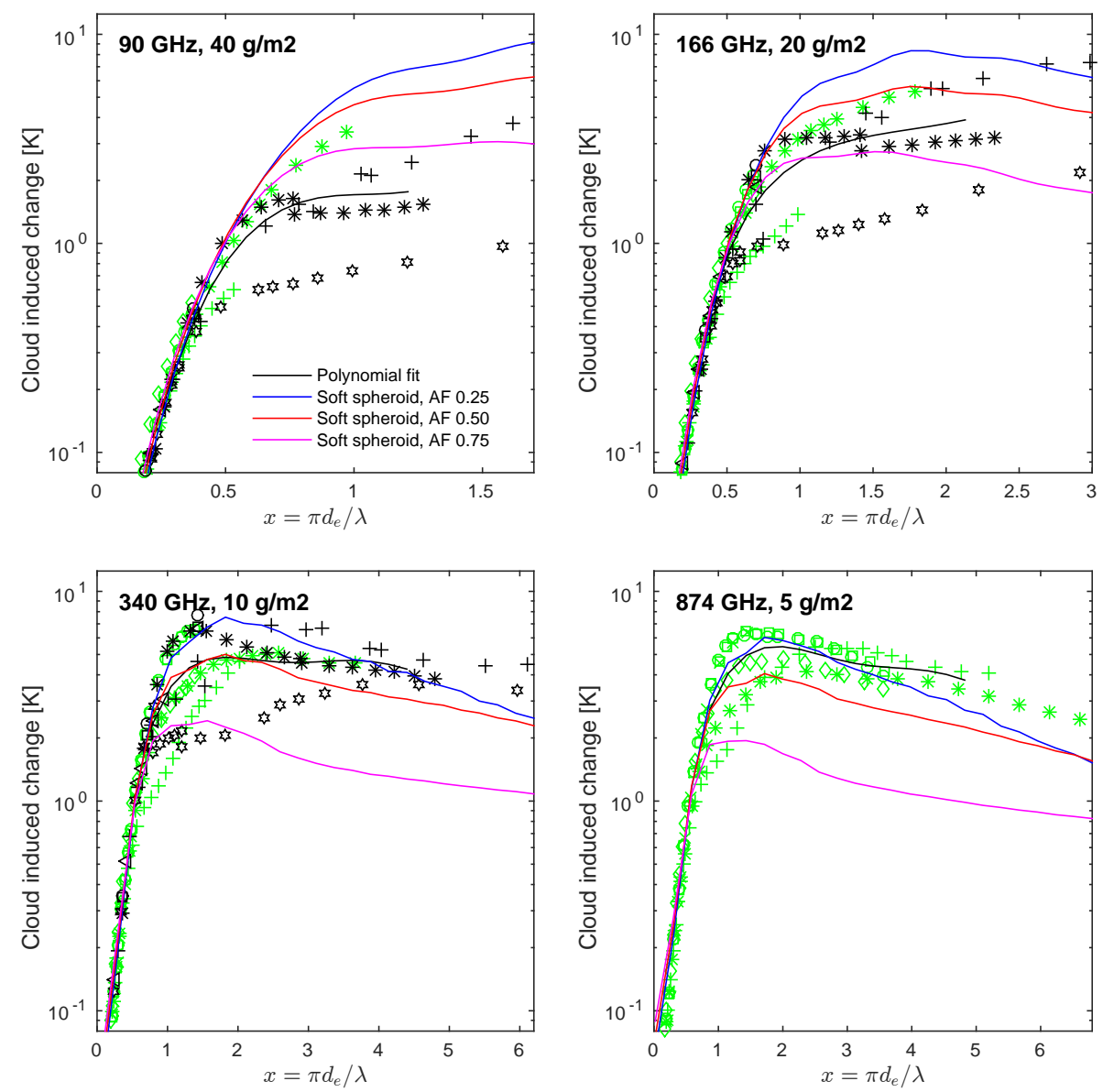

Figure 9. Radiative transfer simulations at different frequencies. General conditions as in Fig. 5, i.e. a $2 \mathrm{~km}$ thick cloud layer at $10 \mathrm{~km}$ is simulated. A single particle type is included in each simulation where the number density was adjusted to obtain the stated ice water paths. The black solid line is a high-order polynomial fit of the DDA-based results, while other lines are results for soft spheroids with constant air fraction (AF).

regions, land/ocean and the different hydrometeor types. Furthermore, a PSD must be assumed for the simulations. The tropical version of the PSD of Field et al. (2007) was found to give the best overall fit with observations among the three PSDs considered. These aspects introduce problems for a clear identification of the best overall proxy particle shape, as discussed in detail by Geer and Baordo (2014).

The final recommendation of Geer and Baordo (2014) is to apply the Liu sector-like snowflake for all classes of both cloud ice and snow. A somewhat better fit could be obtained by some combinations involving the six-bullet rosettes and dendrite snowflake particles, but the improvement was not sufficiently large to motivate a more complicated particle shape model. Our results corroborate the selection of the sector-like snowflake as the general proxy shape particle. This particle type does not stand out in any obvious way; it shows in general intermediate values. In fact, the best match with the polynomial fit of the DDA-based simulations in Fig. 9 (black lines) is given by the sector-like snowflakes for both 90 and $166 \mathrm{GHz}$. A good fit is also obtained by the Liu six-bullet rosette, another particle type that Geer and Baordo (2014) had as a strong candidate. The sector-like snowflake tends to be on the high end for $x$ around 0.7 but on the low side at higher $x$. If these happen to be true biases, they are partly averaged out in PSD-weighted bulk properties.

The Liu sector-like snowflakes exhibit average properties also at $340 \mathrm{GHz}$, above the frequency range considered by Geer and Baordo (2014). The pattern is very similar to the lower two frequencies, with some tendency to "overshoot" around $x=1$. If the upper limit for plates and columns would have been set to a lower value, such as $500 \mu \mathrm{m}$, the sectorlike snowflakes would even have shown outlier behaviour around $x=1$. This results in that for $340 \mathrm{GHz}$ an even better agreement with the polynomial fit is obtained with the Hong aggregates. This particle type of the Hong database is throughout below the fitting line at $874 \mathrm{GHz}$, but this result depends heavily on a strong influence on the polynomial fit of columns and plates with $d_{\mathrm{e}} \approx 500 \mu \mathrm{m}$. The Hong bullet rosette seems not to be a candidate for the role as general 
proxy shape because it has a very low scattering efficiency, around $x \approx 1.5$, which is also reflected in Fig. 9 .

Figures 2 and 3 of Geer and Baordo (2014) complement the figures of this paper by reporting bulk optical properties of the Liu particles as a function of ice mass and frequency. A bit surprising is that the sector-like snowflake is found to have the lowest bulk $g$, seemingly for all frequencies and ice masses. This shape has also the lowest $g$ among the Liu particles in Fig. 3 but only up to $x=1$.

Nevertheless, the SPA spheres applied are found to give very high bulk $g$. Geer and Baordo (2014) explain this as a result of the Mie theory, but according to our Fig. 3 the high $g$ is rather a result of the density assumed. The snow hydrometeor class is set to have a density of $100 \mathrm{~kg} \mathrm{~m}^{-3}$, corresponding to an $\mathrm{AF} \approx 0.9$. We cannot easily judge the exact impact of this relatively high $\mathrm{AF}$ (meaning being close to 1 , not that the true density is misjudged) for several reasons; e.g. Geer and Baordo (2014) used a mixing rule not considered by us. Another complication is that the Field et al. (2007) PSD operates with $d_{\mathrm{m}}$. Hence, also differences in the relationship between particle mass and $d_{\mathrm{m}}$ between the particles affect the data derived by Geer and Baordo (2014). There is a much more intuitive mapping of our findings to bulk properties whether the PSD is based on $d_{\mathrm{e}}$.

Geer and Baordo (2014) analysed only passive observations, while it would be highly beneficial if the representative shape selected could also be applied for radar measurements. Figure 8 indicates that this is the case. The backscattering of the sector-like snowflake follows its pattern for the scattering efficiency (Fig. 7). This shape has a ratio (as defined in discussed figures) above 1 up to a somewhat higher size parameter than the other particles and more pronounced at 35.6 than at $94 \mathrm{GHz}$; besides this, its properties are of average character also with respect to backscattering.

\section{Is using maximum dimension a better option?}

Up to this point, we have compared radiative properties between particles having equal $d_{\mathrm{e}}$ (thus also having the same mass), for reasons discussed in the introduction. The second main measure for the size of individual particles is the maximum dimension, $d_{\mathrm{m}}$. In fact, there are likely more PSDs using $d_{\mathrm{m}}$ than $d_{\mathrm{e}}$. Hence, it is useful to also understand how the radiative properties vary with $d_{\mathrm{m}}$, and such an overview for $183 \mathrm{GHz}$ is given in Fig. 10. This figure was produced as Fig. 3 but with $d_{\mathrm{e}}$ replaced by $d_{\mathrm{m}}$ in the definition of size parameter and absorption and scattering efficiencies.

The panels for asymmetry parameter in Figs. 3 and 10 are quite similar besides the range of $x$ being extended when using $d_{\mathrm{m}}$. However, there are clear differences for both absorption and scattering efficiencies. There is a much more compact relationship between $d_{\mathrm{e}}$ and these radiative properties than what is found for $d_{\mathrm{m}}$. In the case of using $d_{\mathrm{m}}$ as the size measure, relatively compact particles (droxtals, plates, columns and spheres) obtain especially high absorption and scattering efficiencies, while particles having high aspect ratios (dendrite and sector-like snowflakes) exhibit especially low efficiencies. The stronger influence of particle morphology and aspect ratio results in a ratio between highest and lowest efficiency of $\sim 100$ (besides for smallest particles). This is in clear contrast to Fig. 3, where the same ratios are around or below 10 when using $d_{\mathrm{e}}$ (Sect. 3.4).

However, the higher variability in absorption and scattering efficiencies is not directly mapped to the same variability in bulk optical properties, i.e. the optical properties of the distribution as a whole. The reason for this is that particles with high aspect ratio have a lower mass as a function of $d_{\mathrm{m}}$. This aspect deserves careful examination, so we analyse it in the remainder of this section. As a measure for the bulk optical properties we select the scattering extinction coefficient.

As an example of a $d_{\mathrm{m}}$-based PSD we selected the tropical version of the PSD by Field et al. (2007), below denoted as F07. The extinction coefficients were derived with a set-up basically identical to the one described by Geer and Baordo (2014) which also used the F07 PSD: only particles with $d_{\mathrm{m}} \geq 100 \mu \mathrm{m}$ were included (as the PSD does not cover smaller particles), and the PSD was rescaled as described in their Appendix $\mathrm{C}$ to compensate for the truncation in particle size.

An additional aspect of the Field et al. (2007) size distribution is that it uses two additional input parameters, $a$ and $b$. They originate from the common way to express the relationship between $d_{\mathrm{m}}$ and particle mass, $m$, as

$m=a d_{\mathrm{m}}^{b}$.

There are some issues around how to derive $a$ and $b$ for a particle type and how to perform the numerical integrations of the PSD. These issues are described carefully in Appendix B and C of Geer and Baordo (2014) and there is no need to repeat all details. In short, we also selected to derive the $a$ and $b$ parameters by performing a functional fit, but only considering particles with $d_{\mathrm{m}} \geq 100 \mu \mathrm{m}$. A reason to ignore the smaller particles in the fit is that for them Eq. (11) may result in $d_{\mathrm{m}}<d_{\mathrm{e}}$ (corresponding to density higher than the one of solid ice) in cases where $b<3$. As Geer and Baordo (2014) did, we ensured that the bulk mass is preserved by a rescaling of the PSD. This rescaling used the actual particle masses from the DDA database (i.e. assumed $a$ and $b$ were ignored in the rescaling).

However, not all $d_{\mathrm{m}}$-based PSDs take $a$ and $b$ into account. To also investigate the impact of neglecting the dependence on $a$ and $b$, bulk scattering was also derived with F07 and applying fixed $a$ and $b$ values for all particles, namely $a=0.069$ and $b=2$. These values were selected following Wilson and Ballard (1999) and Field et al. (2007).

Since we want to compare $d_{\mathrm{m}}$-based and $d_{\mathrm{e}}$-based bulk extinction coefficients, we also need an example of a $d_{\mathrm{e}^{-}}$ based PSD. For this we selected the PSD by McFarquhar and Heymsfield (1997, below MH97). A comparison between F07 and MH97 is found in Fig. 11, where F07 is rescaled 

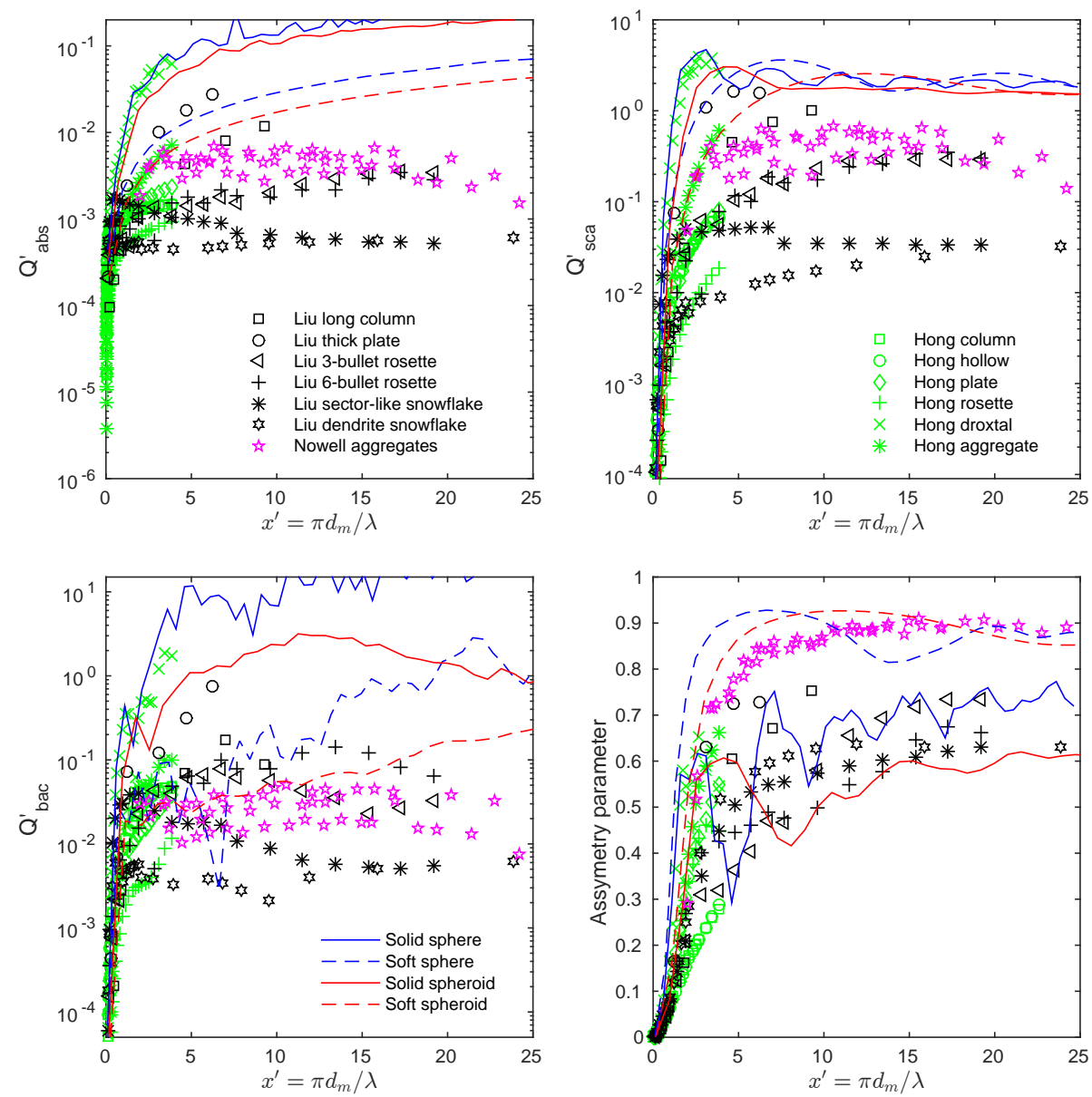

Figure 10. As in Fig. 3 but using the maximum dimension $\left(d_{\mathrm{m}}\right)$ as characteristic size. That is, the size parameter is here defined as $x^{\prime}=$ $\pi d_{\mathrm{m}} / \lambda$ and absorption and scattering efficiencies are defined as $Q^{\prime}=(4 \sigma) /\left(\pi d_{\mathrm{m}}^{2}\right)$. As in Fig. 3, the frequency is $183 \mathrm{GHz}$.

to $d_{\mathrm{e}}$-basis. Two combinations of $a$ and $b$ are considered: the first combination $(0.0015 / 1.55)$ matches the sector-like snowflakes, having the lowest $b$ among all the particles in the Liu database; the second combination (480/3) represents solid spheres and thus also the upper limit of $b$. The rescaling to match specified ice water content has a marginal impact on F07. However, MH97 puts a much larger fraction of the mass below $100 \mu \mathrm{m}$ and the rescaling gives a small but not negligible change; therefore this PSD is displayed both before and after the rescaling. Like F07, MH97 is also a PSD targeting tropical conditions, and the agreement with F07 is relatively high for $a=0.0015, b=1.55$, while for $a=480, b=3$ the two PSDs deviate strongly.

Using the discussed PSDs, bulk scattering extinction coefficients can be calculated by adding up extinction coefficients for individual particles with appropriate weights. Figure 12 shows the results: total extinction coefficients for the two different PSDs, the $d_{\mathrm{m}}$-based F07 at the top and the $d_{\mathrm{e}}$-based MH97 at the bottom. For F07, results for fitted and fixed $a$ and $b$ parameters are shown separately by straight and solid lines respectively.
As the top panel with the F07 results clearly demonstrates, the general pattern seen for the individual particles (Fig. 10) persists in bulk extinction: the more compact particles are at the upper end and the less compact (more "snowflake-like") particles are at the lower end. However, as expected, the ratio between highest and lowest value decreased from $\sim 100$ when considering individual particles to $\sim 10$ when considering bulk extinction. A similar spread in bulk extinction was obtained by Geer and Baordo (2014, see their Figs. 2 and 3).

Still discussing the top panel of Fig. 12, we now turn to the issue of using fitted or fixed $a$ and $b$ parameters for the F07 PSD. For fixed parameters, the extinction obtained for particular shapes is changed, but the spread of the values is roughly maintained. It should here be noted that keeping $a$ and $b$ fixed only has the consequence that the PSD gets the same basic shape for all particles. The rescaling to ensure that specified mass is matched maintains the relative fraction between particles having different $d_{\mathrm{m}}$.

While all the discussion so far related to the top panel of Fig. 12, we will now turn to the bottom panel. It shows that particle shape indeed has a much lower impact on bulk 


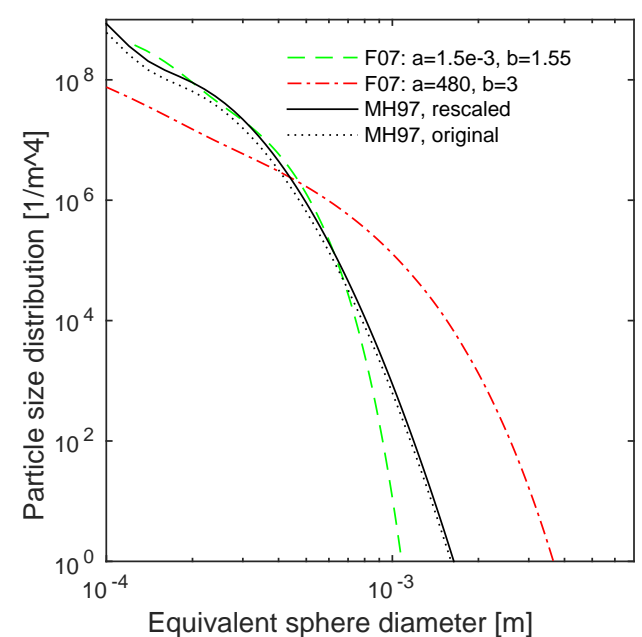

Figure 11. Ice particle size distributions for $0.1 \mathrm{~g} \mathrm{~m}^{-3}$, according to Field et al. (2007, F07) and McFarquhar and Heymsfield (1997, MH97). The F07 PSD is converted from $d_{\mathrm{m}}$ to $d_{\mathrm{e}}$ for two combinations of $a$ and $b$ (Eq. 11).

scattering for the $d_{\mathrm{e}}$-based MH97 PSD compared to the $d_{\mathrm{m}^{-}}$ based F07 PSD. The factor between highest and lowest extinction in case of MH97 is $\sim 2.5$. This cannot be a consequence of MH97 putting the highest weight on completely different particle sizes, as the extinction using MH97 ends up inside the range resulting from F07. Furthermore, the relative variability over the different habits is close to constant with ice water content, for both F07 and MH97, and already Fig. 11 showed that MH97 ends up inside the range covered by F07 when $a$ and $b$ are varied. All in all, F07 and MH97 do a quite similar relative weighting between different particle size ranges. We therefore conclude that the difference in spread seen between upper and lower panel of Fig. 12 is a direct consequence of the deviations revealed by Figs. 3 and 10; i.e. the scattering cross section is more closely linked to $d_{\mathrm{e}}$ than to $d_{\mathrm{m}}$.

The difference between $d_{\mathrm{e}}$ and $d_{\mathrm{m}}$, exemplified by Fig. 12, seems to be of general validity for frequencies up to $\sim$ $200 \mathrm{GHz}$. If anything, the difference increases when going down in frequency (not shown). At higher frequencies a somewhat different pattern is found for the $d_{\mathrm{m}}$ case, as shown in Fig. 13. Here at $340 \mathrm{GHz}$, the spread in extinction of the different DDA particles is overall lower compared to Fig. 12 and is particularly low at high ice water content, where it is even smaller than when using $d_{\mathrm{e}}$. However, the dendrite snowflake particles constitute an exception, which is possibly an indication that this particular shape should perhaps be avoided for higher frequencies. In any case, the deviating results for the dendrite snowflake show that the low spread in extinction between the other particles may be a coincidence, not necessarily indicating that using $d_{\mathrm{m}}$ ensures low uncertainty in extinction for high frequencies and high ice water content. Comparing usage of $d_{\mathrm{e}}$ and $d_{\mathrm{m}}$ at high frequencies
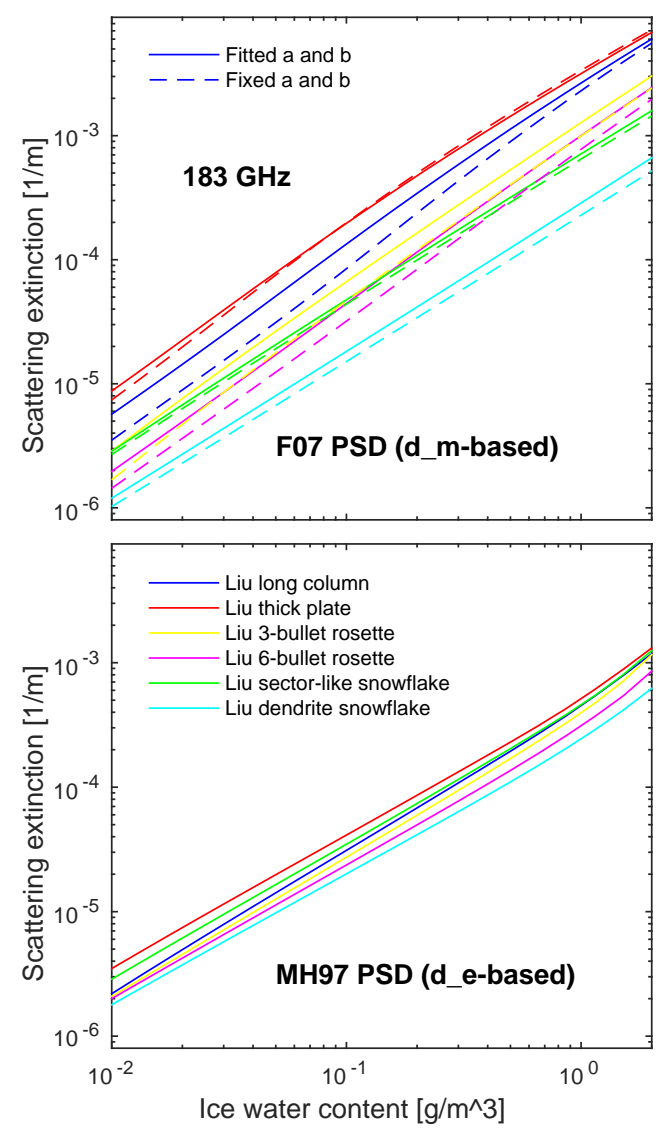

Figure 12. Scattering extinction as a function of ice water content at $183 \mathrm{GHz}$ for some of the particles of the Hong et al. (2009) DDA database. The particle size distribution applied in the upper and lower panels is F07 (Field et al., 2007) and MH97 (McFarquhar and Heymsfield, 1997) respectively. Dashed lines in the upper panel show results for the F07 distribution with fixed $a=0.069$ and $b=2$.

is presently complicated by the fact that the Hong database is limited to $d_{\mathrm{m}} \leq 2 \mathrm{~mm}$ and this size truncation can easily cause artefacts in the comparison.

At the end of this section, we want to briefly mention two more general aspects of the problem of representing bulk particle optical properties. Firstly, our analysis assumed that F07 and MH97 give an equally good representation of the mix of particle sizes. If in situ probes provide better data for either $d_{\mathrm{e}}$ or $d_{\mathrm{m}}$, this should result in higher systematic errors for PSDs based on the more poorly measured particle size.

Secondly, besides possible systematic errors in the PSDs, the variability around average conditions must be considered. For example, it could be the case that there is a lower PSD variation (between locations, day-to-day, etc.) as a function of $d_{\mathrm{m}}$ than as a function of $d_{\mathrm{e}}$. This situation would decrease, or reverse, the advantage of using $d_{\mathrm{e}}$. If the opposite were true, that PSDs tend to be more stable in $d_{\mathrm{e}}$, this would enhance the advantage of selecting $d_{\mathrm{e}}$ in favour of $d_{\mathrm{m}}$. As far 


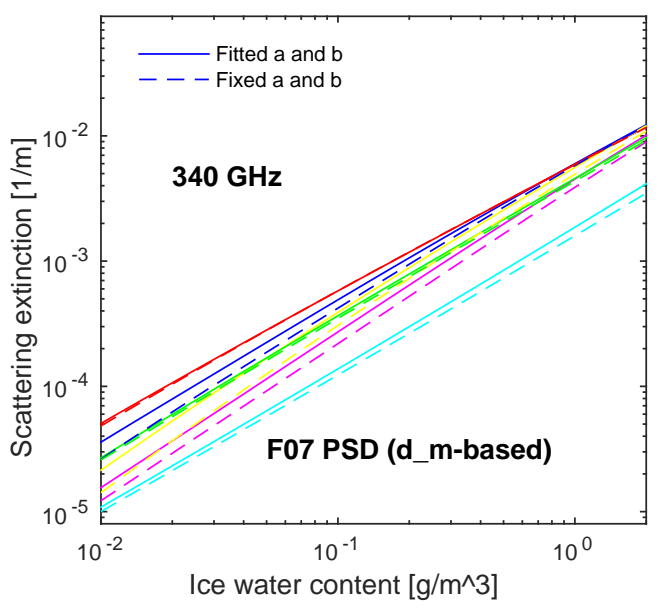

Figure 13. As in Fig. 12 but for $340 \mathrm{GHz}$. The results for MH97 are not shown as they show the same general pattern as in Fig. 12 (just shifted in mean level in the same way as the results for F07).

as we know, this important aspect of PSD variability has not been studied so far.

\section{Summary and conclusions}

We have reviewed the two most established databases of DDA calculations for microwave atmospheric radiative transfer, Liu (2008) and Hong et al. (2009). Nowell et al. (2013) is associated with the Liu database and was also considered. All three databases assume completely random particle orientation. The databases have different frequency coverage: Nowell from 10 to $183 \mathrm{GHz}$, Liu from 3 to $340 \mathrm{GHz}$ and Hong from 90 to $874 \mathrm{GHz}$. Liu is the only database providing data for more than one temperature. Scripts to convert the Hong and Liu data to the format expected by the ARTS forward model can be obtained by contacting the authors.

We noted clear systematic differences in absorption between the Hong and Liu databases. The deviations are explained by the fact that the refractive indices are based on different sources. Hong et al. (2009) used the data from Warren (1984) that now are considered to be outdated. That is, we judge the only easily accessible DDA data above $340 \mathrm{GHz}$ to be inaccurate on particle absorption. In the update of Warren and Brandt (2008), the parameterisation of Mätzler (2006) is recommended for the microwave region, and this is also the source of refractive index used by Liu (2008) and Nowell et al. (2013). Another problematic aspect of the Hong database is the restriction to $d_{\mathrm{m}} \leq 2 \mathrm{~mm}$.

We mainly compared optical properties between particles having the same mass and defined the size parameter $(x)$ accordingly (Eq. 2). For small $x$ below $\approx 0.3$, the variation of absorption and scattering between the particles is about $20 \%$ (1.2 \pm 0.2 in terms of the ratio used in Figs. 4, 7 and 8). Going towards higher $x$, the variation increases most quickly for backscattering, followed by scattering and finally most slowly for absorption. At higher $x$, the ratio between lowest and highest value is $\sim 10, \sim 5$ and $\sim 2.5$ for those three radiative properties respectively. The range in scattering is in general generated by the fact that particles of solid types have comparably high scattering, while shapes of "snow" character result in low scattering. Kim (2006) found that solid spheres are representative up to $x=2.5$ (backscattering not considered and clearly allowing some systematic errors), but we, using a larger set of DDA calculations, find this limit somewhere around $x=0.5$.

We also scrutinised the soft particle approximation. A first conclusion was that the selection of mixing rule can lead to systematic errors at low $x$. A mixing rule giving comparably high refractive index, for given air fraction, is needed to avoid this systematic error. We selected the Maxwell-Garnett with ice as the matrix and air as the inclusion media. With this selection of mixing rule, combined with an air fraction of about 0.25 , SPA is applicable up to about $x \approx 0.5$ across the considered frequency range. This gives for absorption and scattering cross sections a maximum deviation to individual DDA calculations of $\approx 30 \%$.

However, usage of SPA at higher $x$ seems problematic. Each individual property calculated by DDA can likely be reproduced by adjusting the air fraction, but it is in general not possible to achieve a fit with several radiative properties simultaneously. In any case, even fitting a single property, such as backscattering, requires that the air fraction is decreased when moving to higher frequencies. Thus, selecting the air fraction based on some standard density parameterisation can, in the best case, only work in a small frequency range. Our results indicate that this frequency range then is found below $35 \mathrm{GHz}$ as this approach leads to high AFs, passing 0.9 at $d_{\mathrm{m}} \sim 1 \mathrm{~mm}$. At very high frequencies, such as $874 \mathrm{GHz}$, an air fraction of 0.25 could potentially be applied for all particle sizes, but at lower frequencies the air fraction must also be adjusted with size, from about 0.25 at low $x$ (see paragraph above) to a higher value at higher $x$, for example 0.7-0.9 at $90 \mathrm{GHz}$. That is, applying SPA across the microwave region requires a model with a high number of tuning variables to give the air fraction the needed variation with frequency and size, while at the same time the resulting particle densities have no physical basis.

Inspired by Geer and Baordo (2014), we also investigated a second way to represent average radiative properties. The idea is simple: when any of the particles covered by the DDA databases exhibit average properties, use this particle shape to represent true habit mixes. Geer and Baordo (2014) compared the particles of the Liu database using real passive observations, but they were then forced to involve assumptions on particle size distribution and ice mass concentration, while we mainly compared the basic radiative properties directly. However, some radiative transfer calculations were required to assess how differences in scattering cross section and asymmetry parameter combine in simulations of downward-looking passive measurements, but these calcula- 
tions did not involve any additional assumptions. The critical part in our approach is the judgement of how representative the different DDA particles are with respect to the mean conditions in the atmosphere.

Due to the lack of reference data, we selected to not push the analysis too far at this point and discussed only in general terms which particle shapes show overall average properties. It is of course possible to use the same methodology to e.g. select a representative shape separately for "cloud ice" and "snow" or target different cloud types.

Interestingly, Geer and Baordo (2014) and we find that the sector-like snowflake particles, among the shapes found in the Liu database, best represent average properties. This was found valid also for higher frequencies than considered by Geer and Baordo (2014), as well as for application in radar retrievals. For frequencies above $340 \mathrm{GHz}$, where the selection is restricted to the Hong database, an aggregate model appears to be a suitable choice. However, solid conclusions can not yet be drawn, as the number of reference data so far is quite limited. More data of optical properties of aggregate and snow-type particles are needed to get a more robust basis for studies like this. In turn, this requires new algorithms for generating realistic particle models to be used as input to DDA or similar calculation methods. If new databases are created, the limitations of present databases in temperature, particle size and frequencies should be avoided.

Besides the "shape model", we also investigated the representation of particle size. Most importantly, it is demonstrated that there is a much more compact relationship between absorption and scattering properties with mass equivalent diameter $\left(d_{\mathrm{e}}\right)$ than with maximum dimension $\left(d_{\mathrm{m}}\right)$. With the exception of small $x$, the spread of absorption and scattering efficiencies is at least a factor of 10 higher when $d_{\mathrm{m}}$ is used to define the size parameter compared to when using $d_{\mathrm{e}}$. The difference is decreased when summing up individual values to obtain bulk properties, but using a $d_{\mathrm{m}}$-based PSD still gives a higher uncertainty in the extinction for a given ice water content compared to using a $d_{\mathrm{e}}$-based PSD. Below $200 \mathrm{GHz}$, the uncertainties are roughly a factor 10 and 3 for the $d_{\mathrm{m}}$ and $d_{\mathrm{e}}$ case respectively. Scattering extinction at $340 \mathrm{GHz}$ shows a somewhat different pattern and perhaps indicates that the difference between $d_{\mathrm{m}}$ and $d_{\mathrm{e}}$ could vanish at even higher frequencies. In any case, it would be highly beneficial if future in situ measurement campaigns could target to provide PSDs in terms of $d_{\mathrm{e}}$; such measurements seem to be much less frequent than ones of $d_{\mathrm{m}}$.

Finally, we stress that the entire study was performed assuming completely random particle orientation. This is probably the main limitation of the conclusions made above. It can not be ruled out that e.g. the spread of scattering and the difference between using $d_{\mathrm{m}}$ and $d_{\mathrm{e}}$ is highly dependent on particle orientation. That is, a main consideration for future databases of ice hydrometeor optical properties is to make it possible to study the radiative properties when assuming different distributions of horizontal orientation.

\section{The Supplement related to this article is available online at doi:10.5194/amt-8-1913-2015-supplement.}

Acknowledgements. Financial support for this study was provided by the Swedish National Space Board. Furthermore, we want to express our great appreciation to the persons behind the publicly available DDA databases. M. Kahnert, B. Rydberg, M. Brath and A. Geer are thanked for helpful comments.

Edited by: A. Lambert

\section{References}

Austin, R. T., Heymsfield, A. J., and Stephens, G. L.: Retrieval of ice cloud microphysical parameters using the CloudSat millimeter-wave radar and temperature, J. Geophys. Res., 114, D00A23, doi:10.1029/2008JD010049, 2009.

Baran, A. J.: From the single-scattering properties of ice crystals to climate prediction: A way forward, Atmos. Res., 112, 45-69, 2012.

Baran, A. J., Connolly, P. J., Heymsfield, A., and Bansemer, A.: Using in situ estimates of ice water content, volume extinction coefficient, and the total solar optical depth obtained during the tropical ACTIVE campaign to test an ensemble model of cirrus ice crystals, Q. J. Roy. Meteor. Soc., 137, 199-218, 2011.

Bauer, P., Moreau, E., Chevallier, F., and O'Keeffe, U.: Multiplescattering microwave radiative transfer for data assimilation applications, Q. J. Roy. Meteor. Soc., 132, 1259-1281, 2006.

Bennartz, R. and Petty, G. W.: The sensitivity of microwave remote sensing observations of precipitation to ice particle size distributions, J. Appl. Meteorol., 40, 345-364, 2001.

Boukabara, S.-A., Garrett, K., Grassotti, C., Iturbide-Sanchez, F., Chen, W., Jiang, Z., Clough, S. A., Zhan, X., Liang, P., Liu, Q., Islam, T., Zubko, V., and Mims, A.: A physical approach for a simultaneous retrieval of sounding, surface, hydrometeor, and cryospheric parameters from SNPP/ATMS, J. Geophys. Res., 118, 12600-12619, 2013.

Brandes, E. A., Ikeda, K., Zhang, G., Schönhuber, M., and Rasmussen, R. M.: A statistical and physical description of hydrometeor distributions in Colorado snowstorms using a video disdrometer, J. Appl. Meteorol. Clim., 46, 634-650, 2007.

Brown, P. R. and Francis, P. N.: Improved measurements of the ice water content in cirrus using a total-water probe, J. Atmos. Ocean. Tech., 12, 410-414, 1995.

Bruggeman, V. D.: Berechnung verschiedener physikalischer Konstanten von heterogenen Substanzen. I. Dielektrizitätskonstanten und Leitfähigkeiten der Mischkörper aus isotropen Substanzen, Ann. Phys., 416, 636-664, 1935.

Buehler, S. A., Jiménez, C., Evans, K. F., Eriksson, P., Rydberg, B., Heymsfield, A. J., Stubenrauch, C., Lohmann, U., Emde, C., John, V. O., Sreerekha, T. R., and Davis, C. P.: A concept for a satellite mission to measure cloud ice water path and ice particle size, Q. J. Roy. Meteor. Soc., 133, 109-128, doi:10.1002/qj.143, 2007. 
Buehler, S. A., Defer, E., Evans, F., Eliasson, S., Mendrok, J., Eriksson, P., Lee, C., Jiménez, C., Prigent, C., Crewell, S., Kasai, Y., Bennartz, R., and Gasiewski, A. J.: Observing ice clouds in the submillimeter spectral range: the CloudIce mission proposal for ESA's Earth Explorer 8, Atmos. Meas. Tech., 5, 1529-1549, doi:10.5194/amt-5-1529-2012, 2012.

Cotton, R., Field, P., Ulanowski, Z., Kaye, P. H., Hirst, E., Greenaway, R., Crawford, I., Crosier, J., and Dorsey, J.: The effective density of small ice particles obtained from in situ aircraft observations of mid-latitude cirrus, Q. J. Roy. Meteor. Soc., 139, 1923-1934, 2013.

Davis, C. P., Wu, D. L., Emde, C., Jiang, J. H., Cofield, R. E., and Harwood, R. S.: Cirrus Induced Polarization in $122 \mathrm{GHz}$ Aura Microwave Limb Sounder radiances, Geophys. Res. Lett., 32, L14806, doi:10.1029/2005GL022681, 2005.

Debye, P.: Polar molecules, New York : The Chemical Catalog Company, inc, 1929.

Doherty, A., Sreerekha, T., O'Keeffe, U., and English, S.: Ice hydrometeor microphysical assumptions in radiative transfer models at AMSU-B frequencies, Q. J. Roy. Meteor. Soc., 133, 1205$1212,2007$.

Draine, B. T. and Flatau, P. J.: Discrete-dipole approximation for scattering calculations, J. Opt. Soc. Am. A., 11, 1491-1499, 1994.

Draine, B. T. and Flatau, P. J.: User guide for the discrete dipole approximation code DDSCAT (Version 5a10), arXiv preprint astro$\mathrm{ph} / 0008151,2000$.

Emde, C., Buehler, S. A., Davis, C., Eriksson, P., Sreerekha, T. R., and Teichmann, C.: A polarized discrete ordinate scattering model for simulations of limb and nadir longwave measurements in 1-D/3-D spherical atmospheres, J. Geophys. Res., 109, D24207, doi:10.1029/2004JD005140, 2004.

Eriksson, P., Buehler, S. A., Davis, C. P., Emde, C., and Lemke, O.: ARTS, the atmospheric radiative transfer simulator, Version 2, J. Quant. Spectrosc. Ra., 112, 1551-1558, doi:10.1016/j.jqsrt.2011.03.001, 2011a.

Eriksson, P., Rydberg, B., and Buehler, S. A.: On cloud ice induced absorption and polarisation effects in microwave limb sounding, Atmos. Meas. Tech., 4, 1305-1318, doi:10.5194/amt-4-13052011, 2011b.

Evans, K. F. and Stephens, G. L.: Microwave radiative transfer through clouds composed of realistically shaped ice crystals. Part II: Remote sensing of ice clouds, J. Atmos. Sci., 52, 2058-2072, 1995a.

Evans, K. F. and Stephens, G. L.: Microwave radiative transfer through clouds composed of realistically shaped ice crystals. Part I: Single scattering properties, J. Atmos. Sci., 52, 2041-2057, 1995b.

Evans, K. F., Wang, J. R., O'C Starr, D., Heymsfield, G., Li, L., Tian, L., Lawson, R. P., Heymsfield, A. J., and Bansemer, A.: Ice hydrometeor profile retrieval algorithm for high-frequency microwave radiometers: application to the CoSSIR instrument during TC4, Atmos. Meas. Tech., 5, 2277-2306, doi:10.5194/amt5-2277-2012, 2012.

Field, P. R., Heymsfield, A. J., and Bansemer, A.: Snow size distribution parameterization for midlatitude and tropical ice clouds, J. Atmos. Sci., 64, 4346-4365, 2007.

Galligani, V. S., Prigent, C., Defer, E., Jiménez, C., and Eriksson, P.: The impact of the melting layer on the passive microwave cloud scattering signal observed from satellites: A study using TRMM microwave passive and active measurements, J. Geophys. Res., 118, 5667-5678, doi:10.1002/jgrd.50431, 2013.

Garnett, J. M.: Colours in metal glasses, in metallic films, and in metallic solutions. II, Philos. Tr. Roy. Soc. London, 237-288, 1906.

Geer, A. J. and Baordo, F.: Improved scattering radiative transfer for frozen hydrometeors at microwave frequencies, Atmos. Meas. Tech., 7, 1839-1860, doi:10.5194/amt-7-1839-2014, 2014.

Gong, J. and Wu, D. L.: CloudSat-constrained cloud ice water path and cloud top height retrievals from MHS 157 and $183.3 \mathrm{GHz}$ radiances, Atmos. Meas. Tech., 7, 1873-1890, doi:10.5194/amt7-1873-2014, 2014.

Hall, M. P., Goddard, J. W., and Cherry, S. M.: Identification of hydrometeors and other targets by dual-polarization radar, Radio Sci. 19, 132-140, 1984.

Heymsfield, A. J. and Mcfarquhar, G.: Mid-latitude and tropical cirrus - Microphysical properties, in: Cirrus, edited by: D. Lynch, K. Sassen, D. Starr, and G. Stephens, Oxford University Press, 78-101, 2002.

Hogan, R. J., Francis, P., Flentje, H., Illingworth, A., Quante, M., and Pelon, J.: Characteristics of mixed-phase clouds. I: Lidar, radar and aircraft observations from CLARE'98, Q. J. Roy. Meteor. Soc., 129, 2089-2116, 2003.

Hogan, R. J., Tian, L., Brown, P. R., Westbrook, C. D., Heymsfield, A. J., and Eastment, J. D.: Radar scattering from ice aggregates using the horizontally aligned oblate spheroid approximation, J. Appl. Meteorol. Clim., 51, 655-671, doi:10.1175/JAMC-D-11074.1, 2012.

Hong, G.: Parameterization of scattering and absorption properties of nonspherical ice crystals at microwave frequencies, J. Geophys. Res., 112, D11208, doi:10.1029/2006JD008364, 2007.

Hong, G., Yang, P., Baum, B. A., Heymsfield, A. J., Weng, F., Liu, Q., Heygster, G., and Buehler, S. A.: Scattering database in the millimeter and submillimeter wave range of $100-1000 \mathrm{GHz}$ for nonspherical ice particles, J. Geophys. Res., 114, D06201, doi:10.1029/2008JD010451, 2009.

Hufford, G.: A Model For The Complex Permittivity of Ice At Frequencies Below 1 THz, Int. J. Infrared Milli., 12, 677-683, 1991.

Jiang, J. H. and Wu, D. L.: Ice and water permittivities for millimeter and sub-millimeter remote sensing applications, Atmos. Sci. Lett., 5, 146-151, doi:10.1002/as1.77, 2004.

Jiménez, C., Buehler, S. A., Rydberg, B., Eriksson, P., and Evans, K. F.: Performance simulations for a submillimetre wave cloud ice satellite instrument, Q. J. Roy. Meteor. Soc., 133, 129-149, doi:10.1002/qj.134, 2007.

Johnson, B. T., Petty, G. W., and Skofronick-Jackson, G.: Microwave properties of ice-phase hydrometeors for radar and radiometers: Sensitivity to model assumptions, J. Appl. Meteorol. Clim., 51, 2152-2171, 2012.

Kahnert, M., Sandvik, A. D., Biryulina, M., Stamnes, J. J., and Stamnes, K.: Impact of ice particle shape on short-wave radiative forcing: A case study for an arctic ice cloud, J. Quant. Spectrosc. Ra., 109, 1196-1218, 2008.

Kim, M.-J.: Single scattering parameters of randomly oriented snow particles at microwave frequencies, J. Geophys. Res., 111, D14201, doi:10.1029/2005JD006892, 2006.

Kulie, M. S., Bennartz, R., Greenwald, T. J., Chen, Y., and Weng, F.: Uncertainties in microwave properties of frozen precipitation: 
Implications for remote sensing and data assimilation, J. Atmos. Sci., 67, 3471-3487, 2010.

Liao, L., Meneghini, R., Nowell, H. K., and Liu, G.: Scattering computations of snow aggregates from simple geometrical particle models, IEEE J. Sel. Top. Appl., 6, 1409-1417, 2013.

Liebe, H. J., Hufford, G. A., and Cotton, M. G.: Propagation modeling of moist air and suspended water/ice particles at frequencies below $1000 \mathrm{GHz}$, in: AGARD 52nd Specialists' Meeting of the Electromagnetic Wave Propagation Panel, pp. 3-1-3-10, Palma de Mallorca, Spain, 1993.

Liu, G.: Approximation of single scattering properties of ice and snow particles for high microwave frequencies, J. Atmos. Sci., 61, 2441-2456, 2004.

Liu, G.: A database of microwave single-scattering properties for nonspherical ice particles, Bull. Amer. Meteorol. Soc., 89, 1563, doi:10.1175/2008BAMS2486.1, 2008.

Liu, Q., Xue, Y., and Li, C.: Sensor-based clear and cloud radiance calculations in the community radiative transfer model, Appl. Opt., 52, 4981-4990, 2013.

Mätzler, C.: Microwave dielectric properties of ice. Thermal microwave radiation - Applications for remote sensing, Electromagn., Waves Ser., vol. 52, edited by: C. Mätzler, Inst. Eng. Technol., Stevenage, UK, Sect. 5.3, 455-462, 2006.

McFarquhar, G. M. and Heymsfield, A. J.: Parameterization of tropical cirrus ice crystal size distribution and implications for radiative transfer: Results from CEPEX, J. Atmos. Sci., 54, $2187-$ 2200, 1997.

Meirold-Mautner, I., Prigent, C., Defer, E., Pardo, J. R., Chaboureau, J.-P., Pinty, J.-P., Mech, M., and Crewell, S.: Radiative transfer simulations using mesoscale cloud model outputs: Comparisons with passive microwave and infrared satellite observations for midlatitudes, J. Atmos. Sci., 64, 1550-1568, 2007.

Millán, L., Read, W., Kasai, Y., Lambert, A., Livesey, N., Mendrok, J., Sagawa, H., Sano, T., Shiotani, M., and Wu, D. L.: SMILES ice cloud products, J. Geophys. Res., 118, 6468-6477, doi:10.1002/jgrd.50322, 2013.

Mishchenko, M. I., Travis, L. D., and Mackowski, D. W.: T-matrix computation of light scattering by nonspherical particles: A review, J. Quant. Spectrosc. Ra., 55, 535-575, doi:10.1016/00224073(96)00002-7, 1996.

Mishima, O., Klug, D. D., and Whalley, E.: The far-infrared spectrum of ice Ih in the range $8-25 \mathrm{~cm}^{-1}$. Sound waves and difference bands, with application to Saturn's rings, J. Comp. Phys., 78, 6399-6404, doi:10.1063/1.444700, 1983.

Nowell, H., Liu, G., and Honeyager, R.: Modeling the microwave single-scattering properties of aggregate snowflakes, J. Geophys. Res., 118, 7873-7885, doi:10.1002/jgrd.50620, 2013.
Petty, G. W. and Huang, W.: Microwave backscatter and extinction by soft ice spheres and complex snow aggregates, J. Atmos. Sci., 67, 769-787, 2010.

Rydberg, B., Eriksson, P., Buehler, S. A., and Murtagh, D. P.: NonGaussian Bayesian retrieval of tropical upper tropospheric cloud ice and water vapour from Odin-SMR measurements, Atmos. Meas. Tech., 2, 621-637, doi:10.5194/amt-2-621-2009, 2009.

Schmitt, C. G. and Heymsfield, A. J.: Observational quantification of the separation of simple and complex atmospheric ice particles, Geophys. Res. Lett., 41, 1301-1307, 2014.

Sihvola, A.: Mixing rules with complex dielectric coefficients, Journal of Subsurface Sensing Technologies and Applications, 1, 393-425, doi:10.1023/A:1026511515005, 2000.

Skofronick-Jackson, G. M., Gasiewski, A. J., and Wang, J. R.: Influence of microphysical cloud parameterizations on microwave brightness temperatures, 40, 187-196, 2002.

Skofronick-Jackson, G. M., Johnson, B. T., and Munchak, S. J.: Detection thresholds of falling snow from satellite-borne active and passive sensors, 51, 4177-4189, 2013.

Tyynelä, J. and Chandrasekar, V.: Characterizing falling snow using multifrequency dual-polarization measurements, J. Geophys. Res., 119, 8268-8283, doi:10.1002/2013JD021369, 2014.

Warren, S.: Optical constants of ice from the ultraviolet to the microwave, Appl. Opt., 23, 1206-1225, doi:10.1364/AO.23.001206, 1984.

Warren, S. G. and Brandt, R. E.: Optical constants of ice from the ultraviolet to the microwave: A revised compilation, J. Geophys. Res., 113, D14220, doi:10.1029/2007JD009744, 2008.

Wilson, D. R. and Ballard, S. P.: A microphysically based precipitation scheme for the UK Meteorological Office Unified Model, Q. J. Roy. Meteor. Soc., 125, 1607-1636, 1999.

Wu, D. L., Jiang, J. H., Read, W. G., Austin, R. T., Davis, C. P., Lambert, A., Stephens, G. L., Vane, D. G., and Waters, J. W.: Validation of the Aura MLS cloud ice water content measurements, J. Geophys. Res., 113, D15S10, doi:10.1029/2007JD008931, 2008.

Wu, D. L., Lambert, A., Read, W. G., Eriksson, P., and Gong, J.: MLS and CALIOP cloud ice measurements in the upper troposphere: A constraint from microwave on cloud microphysics, 53, 157-165, doi:10.1175/JAMC-D-13-041.1, 2014.

Zhang, C., Lee, K.-S., Zhang, X.-C., Wei, X., and Shen, Y.: Optical constants of ice Ih crystal at terahertz frequencies, Appl. Phys. Lett., 79, 491-493, doi:10.1063/1.1386401, 2001.

Zhao, L. and Weng, F.: Retrieval of ice cloud parameters using the Advanced Microwave Sounding Unit, J. Appl. Meteorol., 41, 384-395, 2002. 